\title{
Brillouin light scattering observation of the transition from the superparamagnetic to the superferromagnetic state in nanogranular $\left(\mathrm{SiO}_{2}\right)$ Co films
}

\author{
A. A. Stashkevich, ${ }^{1, a)}$ Y. Roussigné, ${ }^{1}$ P. Djemia, ${ }^{1}$ D. Billet, ${ }^{1}$ A. I. Stognij ${ }^{2}$ N. N. Novitskii, ${ }^{2}$ \\ G. A. Wurtz, ${ }^{3}$ A. V. Zayats, ${ }^{3}$ G. Viau, ${ }^{4}$ G. Chaboussant, ${ }^{5}$ F. Ott, ${ }^{5}$ S. Gautrot, ${ }^{5}$ \\ M. P. Kostylev, ${ }^{6}$ L. V. Lutsev, ${ }^{7}$ and V. Belotelov ${ }^{8}$ \\ ${ }^{1}$ LPMTM CNRS (UPR 9001), Université Paris 13, 93430 Villetaneuse, France \\ ${ }^{2}$ Institute of Solid State and Semiconductor Physics, National Academy of Sciences of Belarus, 220072 \\ Minsk, Belarus \\ ${ }^{3}$ Centre for Nanostructured Media, IRCEP, The Queen's University of Belfast, Belfast BT7 1NN, United \\ Kingdom \\ ${ }^{4}$ Université de Toulouse, LPCNO/IRSAMC, INSA-UMR CNRS 5215, 31077, Toulouse cedex4, France \\ ${ }^{5}$ Laboratoire Léon Brillouin (LLB-CNRS-CEA) CEA Saclay, 91191 Gif-sur-Yvette Cedex, France \\ ${ }^{6}$ School of Physics-M013, University of Western Australia, 35 Stirling Highway, Crawley, Western Australia \\ 6009, Australia \\ ${ }^{7}$ Research Institute “Ferrite-Domen,” 196084 Saint-Petersburg, Russia \\ ${ }^{8}$ Moscow State University, 119992 Moscow, Russia
}

(Received 5 August 2008; accepted 12 September 2008; published online 7 November 2008)

\begin{abstract}
Evolution of magnetic excitations from purely magnetostatic modes to dipole-exchange spin waves in the Damon-Eshbach geometry in nanocomposite $\left(\mathrm{SiO}_{2}\right)_{100-x} \mathrm{Co}_{x}(50$ at. $\%<x<80$ at. \%) films during a transition from superparamagnetic to superferromagnetic state has been studied by Brillouin light scattering. It has been shown that removal of the degeneracy of backward volume magnetostatic waves due to nonzero exchange constant manifests itself in more pronounced Stokes/ anti-Stokes asymmetry. Accompanying static characterization by means of magnetic force microscopy imaging and grazing incidence small angle neutron scattering has demonstrated the presence of weak stripe domains in the superferromagnetic phase. (c) 2008 American Institute of Physics. [DOI: 10.1063/1.3009339]
\end{abstract}

\section{INTRODUCTION}

The numerous applications of granular films, consisting of magnetic nanoparticles in an insulating matrix, have continuously driven technological and academic research. ${ }^{1-3}$ Their magnetic and electrical properties can be varied over a very wide range by independently modifying the particle sizes and their separation and composition. ${ }^{4}$ Such three dimensional structures are considered as important candidates for the devices based on spin-dependent tunneling.

Static as well as dynamic behavior of such systems is dominated by a trade-off between the long-range dipoledipole interaction (DDI) and the short-range exchange coupling between individual nanoelements, also known as $s u$ perspins. In both cases, it is instrumental in understanding the transition from the superparamagnetic (SPM) state, typical of relatively diluted samples, to the superferromagnetic (SFM) state observed in relatively concentrated samples. However, even in the purely dipole limit, well under the percolation threshold, such a system demonstrates highly nontrivial behavior. The major static features of highly diluted assemblies, i.e., those of isolated magnetic nanoparticles in the absence of interparticle interactions, are well described by the classical model of to $\mathrm{Neel}^{5}$ and Brown, ${ }^{6}$ which provides relatively simple and clear physical picture. The behavior of isolated particles, determined in this ap-

${ }^{a)}$ Electronic mail: as@1pmtm.univ-paris13.fr. proximation by the thermally activated relaxation between the potential wells of the anisotropy energy, are well verified by the magnetization and low-frequency susceptibility measurements in a wide temperature range for the samples "prepared" according to different scenarios: zero-field cooling (ZFC), positive low field cooling, positive high field cooling, negative low field cooling, or negative high field cooling. ${ }^{\text {? }}$

Introduction of DDIs, however weak, leads to serious theoretical complications due to their long range nature. ${ }^{8}$ Assemblies of sparse weakly interacting magnetic dipoles can be regarded as "frozen ferrofluids." Experimental studies, via Mössbauer spectroscopy, ac susceptibility, and ZFC magnetic relaxation measurements in $\mathrm{Fe}-\mathrm{C}$ frozen ferrofluids have shown that even in highly dilute samples collective magnetic dynamics and critical slowing down at a finite temperature take place. ${ }^{9}$ Moreover, similar experiments performed in more concentrated ferrofluids, in which the dipolar interaction energy dominates over other contributions to the total energy, have shown that such particle systems exhibit nonequilibrium dynamics with apparent similarities to the nonequilibrium dynamics of spin glasses. ${ }^{10}$ These effects, however, disappear in moderate external magnetic fields. ${ }^{11}$ The important issue of the influence of ordering in $\mathrm{Co} / \mathrm{Al}_{2} \mathrm{O}_{3}$ multilayers has been addressed in Ref. 12, while the influence of the antiferromagnetic $\mathrm{CoO}$ shell in such structures has been investigated in Ref. 13. It should be noted that in neither case has the percolation threshold been approached 
and no interparticle exchange coupling was possible; hence all effects observed were of purely dipole nature.

However, the physical picture of collective magnetic behavior is incomplete without taking into account the exchange interaction. In this respect, much original research has been done on a particular variety of dense nanocomposite materials known as discontinuous metal-insulator multilayers comprised of $\mathrm{CoFe}$ nanoparticles in an $\mathrm{Al}_{2} \mathrm{O}_{3}$ matrix. ${ }^{14-17}$ The specificity of these structures lies in the fact that while the in-plane structure is rather disordered (the dispersion of the nanoparticle size is large and particle in-plane spacing varies considerably), the interlayer distance is controlled with high precision. Such dense multilayers have demonstrated the full spectrum of possible types of behavior. The transition from superparamagnetism ${ }^{16}$ to superferromagnetism ${ }^{15,17}$ via the superspin glass ${ }^{15,16}$ state has been implemented by simply varying the particle diameter to interlayer distance ratio. As in the case of ferrofluids, the characterization of the investigated structures has been based on the static behavior and slow dynamics, experimental methods including $\mathrm{x}$-ray photoemission electron microscopy, magneto-optical Kerr microscopy, field cooling (FC), ZFC, and ac susceptibility measurements in a wide range of temperatures.

Another group of papers, falling in a far less numerous category, has been dedicated to fast dynamics, more precisely to microwave properties of saturated magnetic nanogranular materials in the gigahertz range. ${ }^{18-20}$ Collective high-frequency dynamics of saturated granular structures manifests itself in two types of magnetic excitations propagating in the bulk of such films: purely dipole magnetostatic waves in the case of low concentration of magnetic particles and dipole-exchange spin waves (SWs) in concentrated samples. The investigations of the ferromagnetic resonance (FMR) in magnetically heterogeneous $\mathrm{Fe}-\mathrm{SiO}_{2}$ composite ultrathin films in the vicinity of the percolation threshold have demonstrated the presence of spin-wave resonances (SWRs) due to a nonzero effective exchange constant. The FMR lines were closely spaced, thus indicating very small values of the effective exchange constant in the structure. Moreover, their equidistant spacing pointed to a very high degree of magnetic inhomogeneity of the thin samples investigated.

The FMR technique, based on the resonant amplification of the exciting high-frequency electromagnetic field, is extremely sensitive. However, FMR characterization of collective magnetic excitations is incomplete, being limited to spatially uniform external microwave pumping. This means that only SWs with the in-plane wave number $k$ equal to zero are generated and investigated. In this respect it should be noted that the Brillouin light scattering (BLS) spectroscopy, although very time consuming, provides very important additional degrees of freedom in the investigation of SW behavior. It relies on the inelastic scattering of light by thermally excited SWs through the induced modulation of the optical constants of the medium via the magneto-optic interaction. First of all, it covers the SW excitations with the wave numbers in the extremely wide range $3 \times 10^{3} \mathrm{~cm}^{-1}<k<2$ $\times 10^{5} \mathrm{~cm}^{-1}$, which allows tracing the full $\mathrm{SW}$ dispersion curve even in relatively thin samples. Moreover, making use of the magnons, excited by stochastic thermal mechanisms, it provides access to the full spectrum of SW magnetic excitations, contrary to the FMR techniques. In the latter case, only the SWR modes forming nonzero overlap integrals with the external homogeneous microwave field can be excited. This excludes, for example, any SWR in films with free surface spins. Besides, all the antisymmetric SWR modes cannot be excited under any conditions, except nonsymmetric surface pinning.

Not surprisingly, the BLS technique has proved to be one of the most powerful methods for the investigation of the physical properties of structured magnetic multilayers. ${ }^{21-23}$ Extensive research carried out in this domain, motivated by important applications to Magnetic Random Access Memory (MRAM) and reading magnetic heads, has lead to some significant results, both experimental and theoretical. Due to a simpler one-dimensional (arrays of stripes) or twodimensional (2D) (arrays of dots) geometry of these systems, more physical insight can be ensured, allowing better understanding of physical phenomena induced by dipolar interactions. Typically, the elements of such arrays are of micron or submicron size carrying individual in-plane resonances of DDI dominated magnetostatic modes. Thus the wave numbers $k$ proportional to multiples of the inverse values of the in-plane dimensions of the array elements are imposed. These wave numbers lie exactly in the $k$ range most suitable for BLS studies. In most cases the interelement DDIs are negligible and no traces of collective behavior are observed. However, if the interelement spacing is minimized, being of order of tens of nanometers, the features typical of magnonic crystal behavior, ${ }^{24}$ such as formation of Brillouin zones predicted earlier theoretically, ${ }^{25}$ are revealed by BLS studies. ${ }^{26}$

The specificity of the case addressed in this paper lies in the fact that a monolayer structure is comprised of spherical nanoelements whose size is much less than the SW wavelength $\lambda_{\mathrm{SW}}$. The minimum value of $\lambda_{\mathrm{SW}}$ involved in the BLS corresponds to $\lambda_{\text {light }} / 2$, which amounts, typically, to $250 \mathrm{~nm}$. At the same time, the size of nanograins in a composite material normally does not exceed $6 \mathrm{~nm}$, which ensures the collective character of the microwave dynamics of the system. In the present paper, the transition from DDI dominated SPM magnetic wave dynamics to SFM wave behavior, strongly influenced by the interparticle exchange interaction, will be investigated. To this end, the evolution of the dispersion characteristics, measured by the BLS technique, from the purely magnetostatic-type (SPM) to the dipole-exchangetype (SFM) will be analyzed, aided by numerical simulations. The latter will include computer simulations of the fine structure of the BLS Stokes and anti-Stokes lines, based on the fluctuation-dissipation theorem (FDT) and the calculations of the SW spectra making use of the magnetic Green's function approach. Static characterization of the samples will include superconducting quantum interference device (SQUID), magnetic force microscopy (MFM)/atomic force microscopy (AFM), focused ion beam (FIB)/transmission electron microscopy (TEM) imaging, $\mathrm{x}$-ray diffraction, and scattering of polarized neutrons. 
TABLE I. Basic parameters of composite samples.

\begin{tabular}{lcccc}
\hline \hline & & & & \\
Sample number & 1.1 & 1.2 & 1.3 & 1.4 \\
Co concentration (at. \%) & 80 & 73 & 60 & 50 \\
Thickness (nm) & 600 & 560 & 520 & 450 \\
Particle spacing (nm) & 0.1 & 0.7 & 1.9 & 3.0 \\
\hline \hline
\end{tabular}

\section{SAMPLE PREPARATION AND CHARACTERIZATION}

The experiments were performed on samples of amorphous silicon dioxide films containing cobalt nanoparticles grown on a gallium arsenide substrate, designated as $\left(\mathrm{SiO}_{2}\right)_{100-x} \mathrm{Co}_{x}$ or $\mathrm{SiO}_{2}(\mathrm{Co}) . n$-GaAs substrates are of the (100)-orientation type. The GaAs substrate thickness is 0.4 $\mathrm{mm}$. Prior to the deposition process, substrates were polished by a low-energy oxygen ion beam. Methods of ion beam polishing are described in Refs. 27 and 28. On the ion beam processed surface, the maximum roughness height did not exceed $0.5 \mathrm{~nm}$.

The $\mathrm{SiO}_{2}(\mathrm{Co})$ films were grown on $\mathrm{GaAs}$ substrates by ion beam cosputtering of composite quartz and cobalt targets. The concentration of cobalt nanoparticles in the silicon dioxide deposit was varied by changing the ratio of cobalt and quartz target areas. The films were deposited onto GaAs substrates heated to $200{ }^{\circ} \mathrm{C}$. The parameters of the four samples studied, including the relative content of cobalt $x$, the $\mathrm{SiO}_{2}(\mathrm{Co})$ film thickness $h$, and interparticle spacing $d$ assessed from $x$ via the calculation of the volume concentration of Co, are summarized in Table I. The film composition was determined by $\mathrm{x}$-ray analysis. It has been doublechecked by Rutherford backscattering. The discrepancy between the two figures does not exceed a few percents.

To estimate the actual size of the particles, we have employed three independent techniques: low-angle x-ray scattering, neutron scattering, and TEM imaging. Using a FEI FIB200TEM nanomachining system, a parallel-sided lamella ( $300 \mathrm{~nm}$ ) was milled to prepare the cross-section cut. The cross section was imaged with a JEOL 2010 (200 kV) TEM. According to the low-angle $\mathrm{x}$-ray scattering measurements, the average size of cobalt particles increases with $x$ : from 2.7 $\mathrm{nm}$ at $x=50$ at. $\%$ to $3.5 \mathrm{~nm}$ at $x=60$ at. \%. However, the values retrieved from high-resolution TEM images (see Fig. 1) are slightly greater, being of the order of 5-6 $\mathrm{nm}$ for $x$ $=60$ at. $\%$.

Static magnetic properties of the samples were characterized by MFM and SQUID. Figure 2 gives AFM/MFM images of samples 1.1, 1.2, and 1.3, i.e., as a function of $\mathrm{Co}$ content. As is clearly seen in Fig. 2(a), a classic maze domain pattern is formed in the first sample with the highest $\mathrm{Co}$ concentration $x=80 \%$. Just as in the case of continuous ferromagnetic samples, its structure demonstrates a tendency to transform into a stripelike geometry after exposure to an attenuating low-frequency magnetic field [see Fig. 2(b)]. For low concentrations, the domain structure is less pronounced. For $x=73 \%$ [sample 1.2, Fig. 2(c)] it is closer to the bubble geometry and for $x=60 \%$ it is barely visible [sample 1.3, Fig. $2(\mathrm{~d})]$.

To reliably identify the actual magnetic state of the

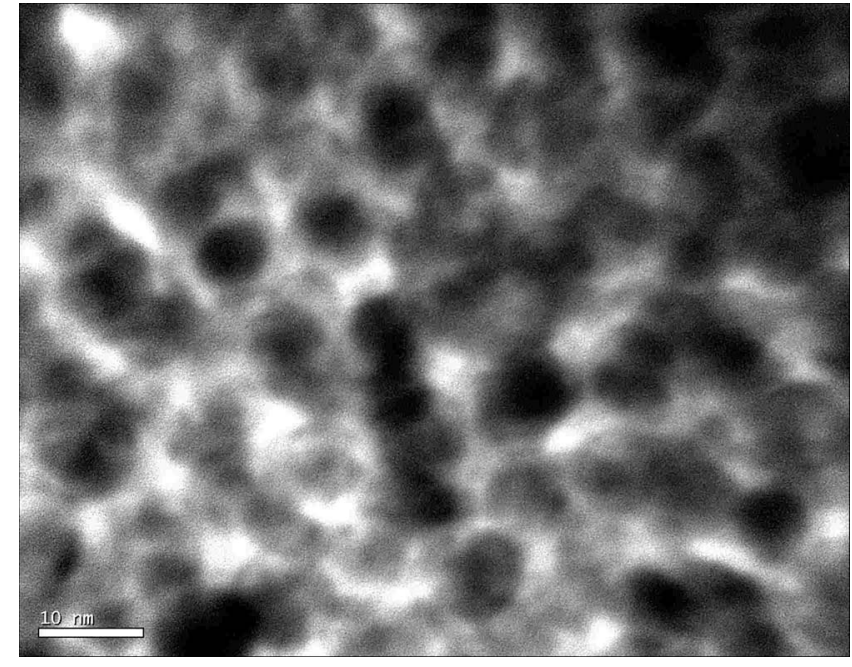

FIG. 1. High resolution TEM image from a cut of sample 1.3.

$\mathrm{Co}-\mathrm{SiO}_{2}$ films SQUID (Quantum Design MPMS-5S) measurements have been undertaken. The temperature dependence of the magnetization of the films was measured in the range of 5-300 K with an applied field of $200 \mathrm{Oe}$, both in the ZFC and FC modes. The measurement time was typically 30 $\mathrm{s}$ for each temperature.

The results for two extreme cases are given in Fig. 3. Figure 3(a) corresponds to the most concentrated sample 1.1 $(x=80 \%)$ and Fig. 3(b) to the least concentrated sample 1.4 $(x=50 \%)$. In the first case, the magnetization is quite constant over the whole temperature range. Moreover, no marked irreversibility between the magnetization curves measured in FC and ZFC was observed (Fig. 3). These results show a ferromagnetic behavior of the film in the whole temperature range studied. On the contrary, the FC-ZFC curves of sample 1.4 show a SPM behavior with a blocking temperature of $100 \mathrm{~K}$ [Fig. 3(b)].

The field dependence of magnetization has been measured at $300 \mathrm{~K}$ with the applied field parallel and perpendicular to the films (Fig. 4). The concentrated sample 1.1 was fully saturated for an applied field of 2000 Oe for the parallel orientation [Fig. 4(a)]. At high field the diamagnetic contribution of the substrate decreases the total magnetization and can be corrected with a linear contribution. The saturation magnetization of the film $\left(760 \mathrm{emu} \mathrm{cm}^{-3} ; 4 \pi M_{s}=9.55\right.$ $\times 10^{3} \mathrm{G}$ ) with $H$ parallel is consistent with the atomic concentration of the cobalt particles within $\mathrm{SiO}_{2}$ of $80 \%$ and a film thickness of $600 \mathrm{~nm}$. With the field perpendicular to the film, the value of 9000 Oe was necessary to reach the saturation, this value is consistent with the $4 \pi M_{s}$ value of the film. In the following we will use this magnitude as the effective value of the saturation magnetization of the composite material. In the case of the diluted SPM sample 1.4, high magnetic fields, of the order of $10 \mathrm{kOe}$, are required to fully saturate the film in both parallel and perpendicular geometries [Fig. 4(b)]. At the same time, the slope of the "parallel curve" is clearly steeper than that of the "perpendicular curve." In sample 1.1 at $300 \mathrm{~K}$ the coercivity and remanence are very small either in the parallel or in the perpendicular orientation. At $5 \mathrm{~K}$ in the parallel orientation the coercivity is 


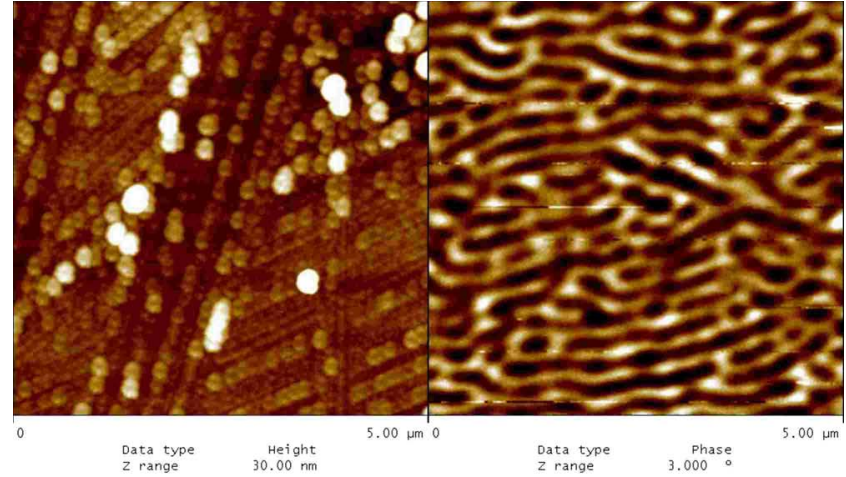

(a)

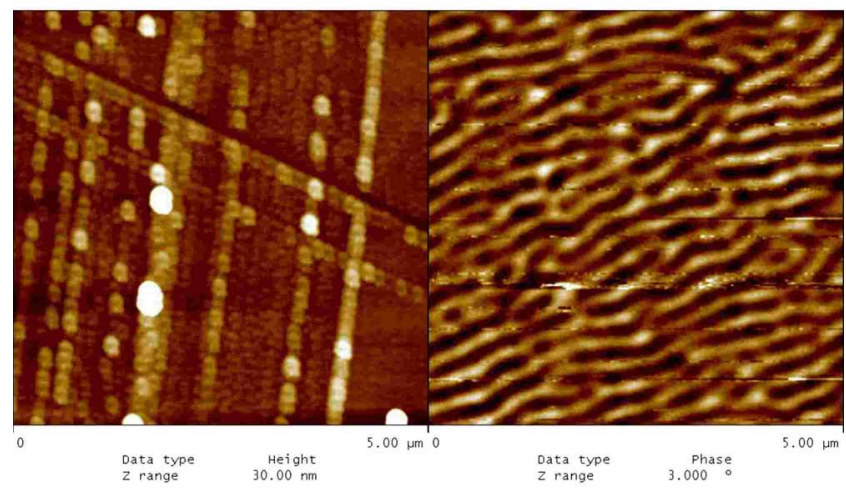

coinsio2. 002 ing
chamo desexcitation 400 oe

(b)

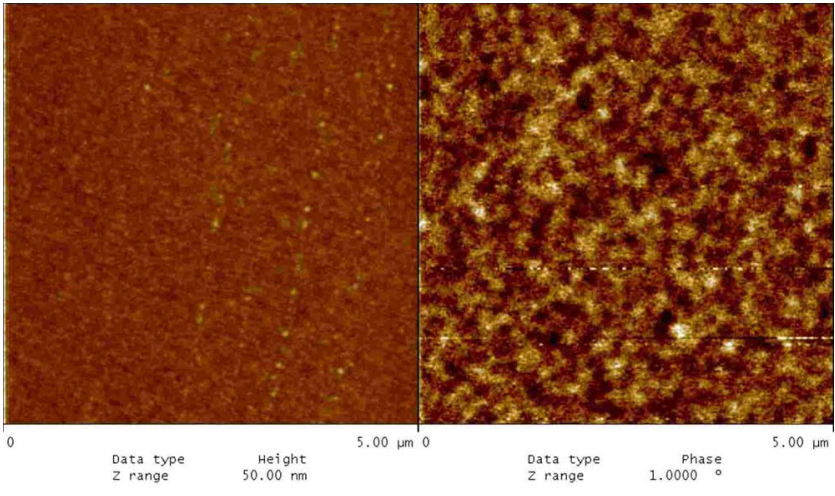

sio2co73.001

(c)

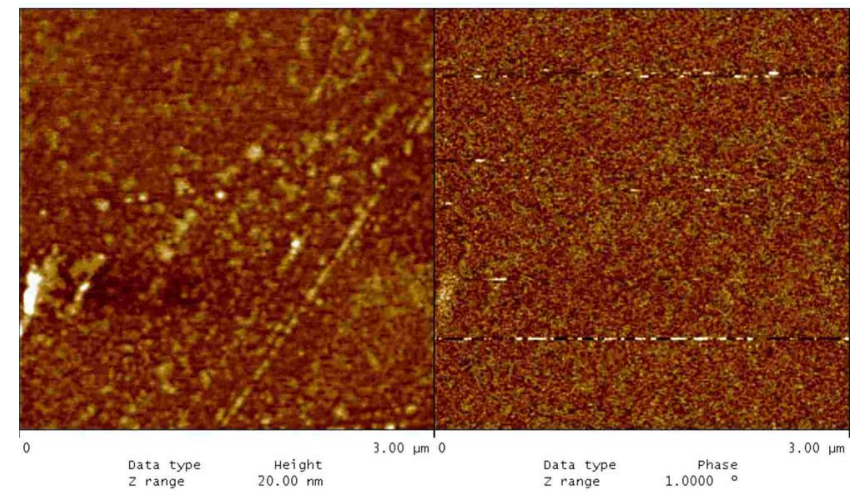

(d)

FIG. 2. (Color online) AFM (on the left hand side) and MFM (on the right hand side) images of (a) sample $1.1(x=80 \%)$, (b) same sample after exposure to an attenuating low-frequency magnetic field, (c) sample $1.2(x=73 \%)$, and (d) sample $1.3(x=60 \%)$.

found to be equal to $375 \mathrm{Oe}$ [Fig. 5(a)]. The diluted sample 1.4 shows a ferromagnetic behavior under the blocking temperature and a marked coercitivity $\left(H_{c}=825 \mathrm{Oe}\right)$ is observed at $5 \mathrm{~K}$ [Fig. 5(b)].

According to TEM/FIB cuts the samples were not very homogeneous. In order to check overall characteristics, specular and off-specular neutron reflectivity measurements have been performed using the spectrometer Papyrus at the laboratoire Léon Brillouin (LLB) (CEA-Saclay, France). The mean size of the Co particles was estimated by small angle $\mathrm{x}$-ray scattering to be around $3 \mathrm{~nm}$ with a slight dependence on the Co concentration. This is in good agreement with the data provided by low-angle $\mathrm{x}$-ray scattering, although slightly underestimates the size with respect to the TEM/FIB images.

Papyrus is the multipurpose [small angle neutron scattering (SANS) and reflectivity] instrument operating at a fixed neutron wavelength $(\lambda=0.8 \mathrm{~nm})$ with a $2 \mathrm{D} X Y$ detector (grid of $128 \times 128$ wires with $5 \mathrm{~mm}$ size) placed $4 \mathrm{~m}$ behind the sample position.

Figure 6(a) shows the 2D pattern obtained with an incident angle $\theta=0.5^{\circ}$ at $T=300 \mathrm{~K}$. The first information is obtained from the specular reflectivity $\left(\theta_{i}=\theta_{f}\right)$ with a $Q_{z}$ value given by $Q_{z}=(4 \pi / \lambda) \sin (\theta)$. Like for $\mathrm{x}$ rays, recording the reflectivity curve as a function of incident angle enables the determination of the depth nuclear composition profile of the sample. The neutron reflectivity curve as a function of the angle of incidence is shown in Fig. 6(b). The critical angle $\theta_{c}$ for total reflection is estimated to be $0.35^{\circ}-0.40^{\circ}$. For continuous media the critical angle is given by

$$
\sin \left(\theta_{c}\right)=\sqrt{\frac{\rho b}{\pi}} \lambda,
$$

where $\rho$ is the scattering object density, $b$ is the nuclear scattering length, and $\lambda$ is the neutron wavelength. With $\theta_{c}$ $\approx 0.375^{\circ}$, one obtains $\rho b \approx 2.1 \times 10^{10} \mathrm{~cm}^{-1}$. The experimental value is not far from pure Cobalt $\left(2.2 \times 10^{10} \mathrm{~cm}^{-1}\right)$ but substantially higher than pure $\mathrm{SiO}_{2}\left(3.96 \times 10^{10} \mathrm{~cm}^{-1}\right)$ or even GaAs $\left(3.0 \times 10^{10} \mathrm{~cm}^{-1}\right)$. Fitting the reflectivity curve of heterogeneous materials is more complex and requires one to take into account the various layers in the material. ${ }^{29}$ The best fit, shown as red line in Fig. 6(b), is obtained for $\rho b$ values comprised between $1.5 \times 10^{10}$ and $2.5 \times 10^{10} \mathrm{~cm}^{-1}$, in general agreement with the $\mathrm{Co} / \mathrm{SiO}_{2}$ composition. However, it was not possible to include the particle sizes or shapes in the calculation. Alternatively, it is possible to model the $\mathrm{Co} / \mathrm{SiO}_{2}$ layer by considering a stack of alternating pure $\mathrm{Co}$ and $\mathrm{SiO}_{2}$ layers while using their nominal $\rho b$ values as fixed parameters. Simulating the $\mathrm{Co} / \mathrm{SiO}_{2}$ layer with such thin alternate layers brings qualitatively good results when choosing an averaged thickness of 7.4-8.0 nm for each layer. The best fit result [blue line in Fig. 6(b)] overestimates the critical angle by $0.05^{\circ}$ only. 

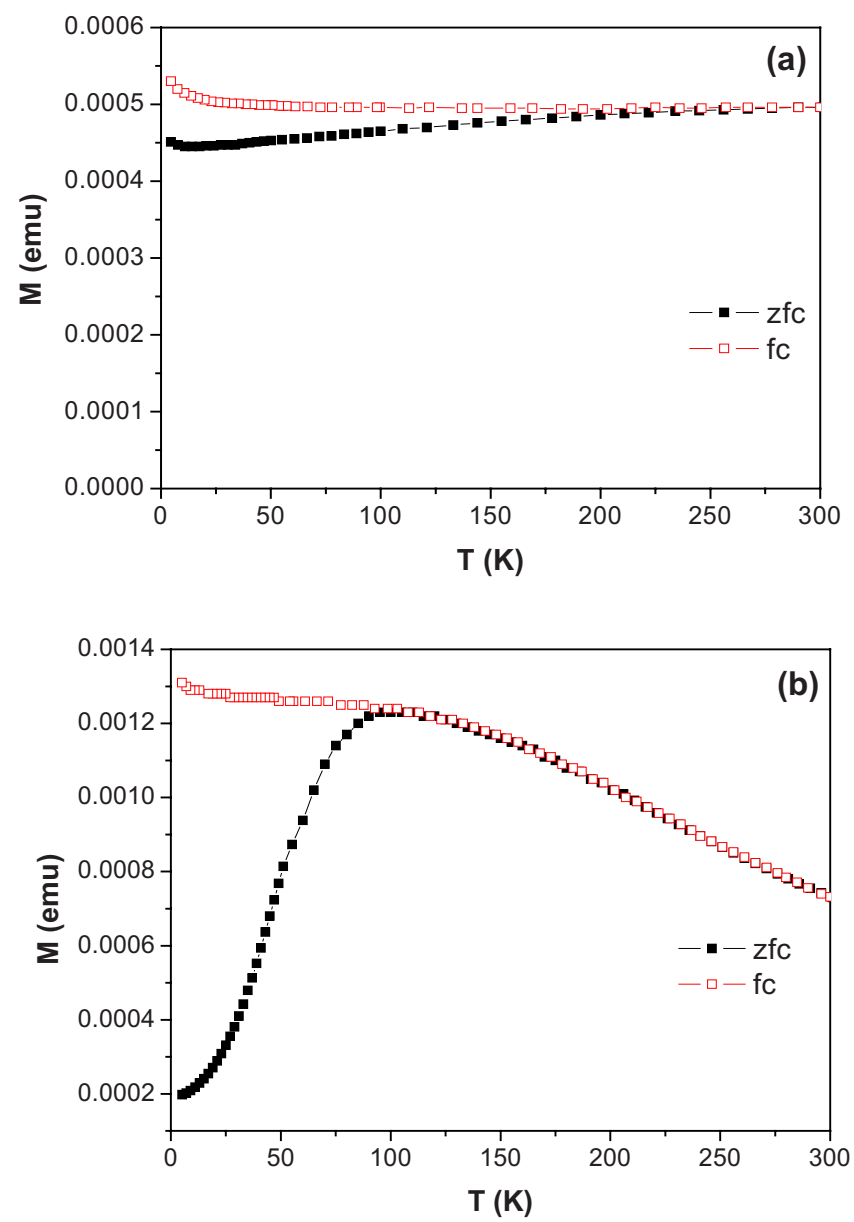

FIG. 3. (Color online) Temperature dependence of the magnetization of Co-SiO2/AsGa films measured in ZFC (full symbols) and FC (open symbols) modes under an applied field $H=200$ Oe: (a) sample $1.1(x$ $=80$ at. $\%)$ and (b) sample $1.4(x=50$ at. \%).

Grazing incidence SANS (GISANS) brings information about the lateral structures and correlations of surfaces and near-surface layers. Above the critical field, an evanescent neutron wave forms at and below the surface; diffraction effects may then occur if periodic structures or nanometric domains are present. As shown in Fig. 7(a), GISANS signal appears as an off-specular signal perpendicular to the neutron direction [vertical direction in Fig. 7(a)]. It is the maximum intensity and width for incident angles near the critical angle $\theta_{c}=0.35^{\circ}-0.4^{\circ}$. For values above $\theta_{c}$, the GISANS signal rapidly fades away. Figure 7(b) shows two cuts along the GISANS signal, one corresponding to the total reflection range (circles) and the other just above the critical field where the GISANS intensity is theoretically expected to be strongest. On both sides of the specular spot, the extended scattering observed at $0.45^{\circ}$ is reminiscent of large domain formation at the surface or near the surface. Fitting the extra scattering by two Gaussians peaked symmetrically from the specular position at $Q= \pm 0.05 \mathrm{~nm}^{-1}$, it is possible to infer the existence of large domains (125 $\mathrm{nm}$ in average) with a wide size distribution, as evidenced by the large width $(\Gamma$ $\approx 0.06 \mathrm{~nm}^{-1}$ ). The formation of domains in the size range of 50-200 nm of the $\mathrm{Co} / \mathrm{SiO}_{2}$ layer, implying a distribution of $\mathrm{Co}$ and $\mathrm{SiO}_{2}$ in the matrix, can thus be invoked to account for the observed GISANS data. The domain size thus esti-
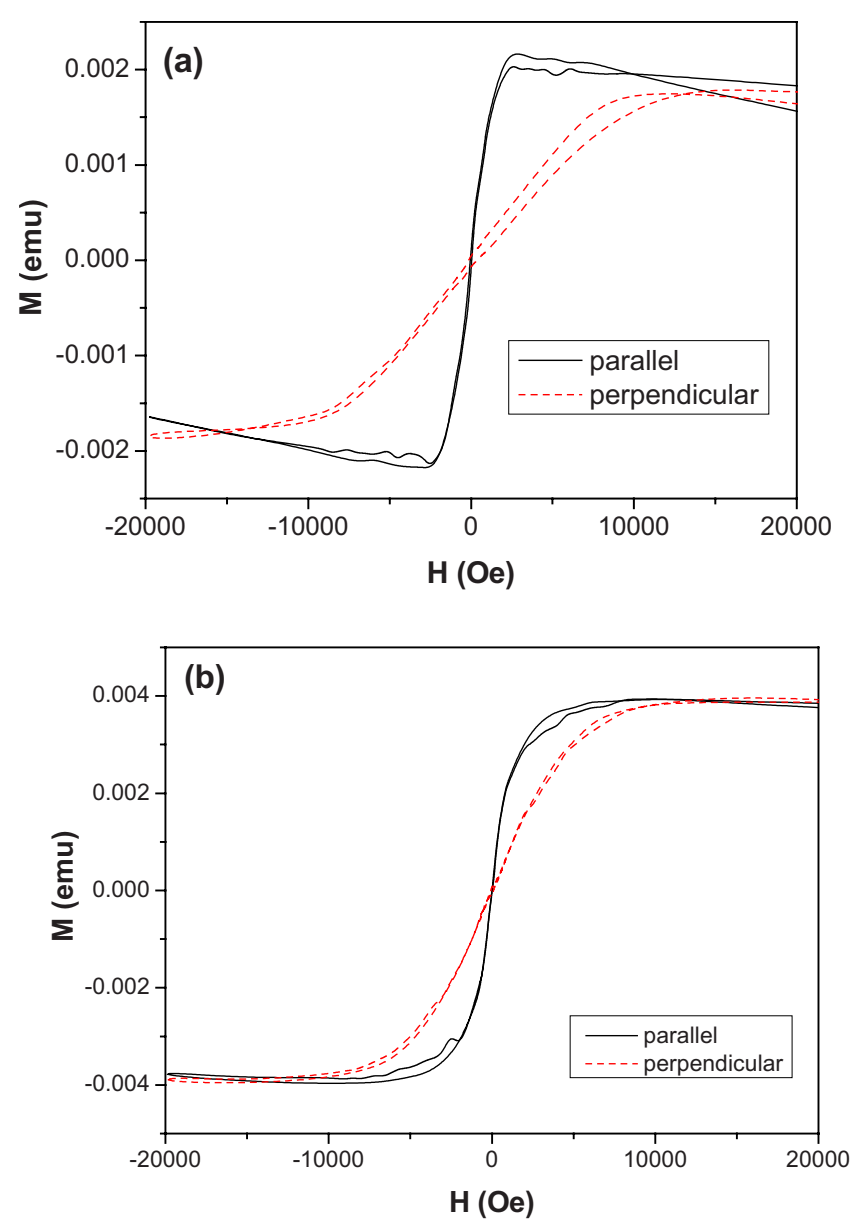

FIG. 4. (Color online) Magnetization curves at $300 \mathrm{~K}$ of Co-SiO2/AsGa films with the applied field parallel (full lines) and perpendicular to the film (dashed lines): (a) sample $1.1 \quad(x=80$ at. \%) and (b) sample $1.4(x$ $=50$ at. $\%$.

mated agrees well with the domains in Fig. 2. Similar domain structures, observed by neutron scattering, have been reported in Ref. 4.

\section{EXPERIMENTAL RESULTS AND DISCUSSION}

The BLS measurements ( $p-s$ scattering) were carried out in the Damon-Eshbach (DE) geometry. The saturating magnetic $\vec{H}$ field was applied parallel to the plane of the film and the direction of propagation of a SW was normal to $\vec{H}$.

Light from a single-mode $\mathrm{Ar}^{+}$laser using a power of 350 $\mathrm{mW}$ at wavelength $\lambda$ of $514 \mathrm{~nm}$ was focused onto the sample and the frequency spectrum of the backscattered light was analyzed using a computer controlled Sandercock-type (2 $\times 3)$-pass tandem Fabry-Pérot interferometer. The value of the wave number $k$ was changed by varying the angle of incident light $\theta$ measured against the surface normal to the sample,

$$
k=(4 \pi / \lambda) \sin \theta .
$$

Cross polarizations between the incident and scattered beams were used in order to practically suppress the light scattered by phonons.

For the first series of BLS measurements we have selected the most concentrated sample 1.1. To ensure the maxi- 

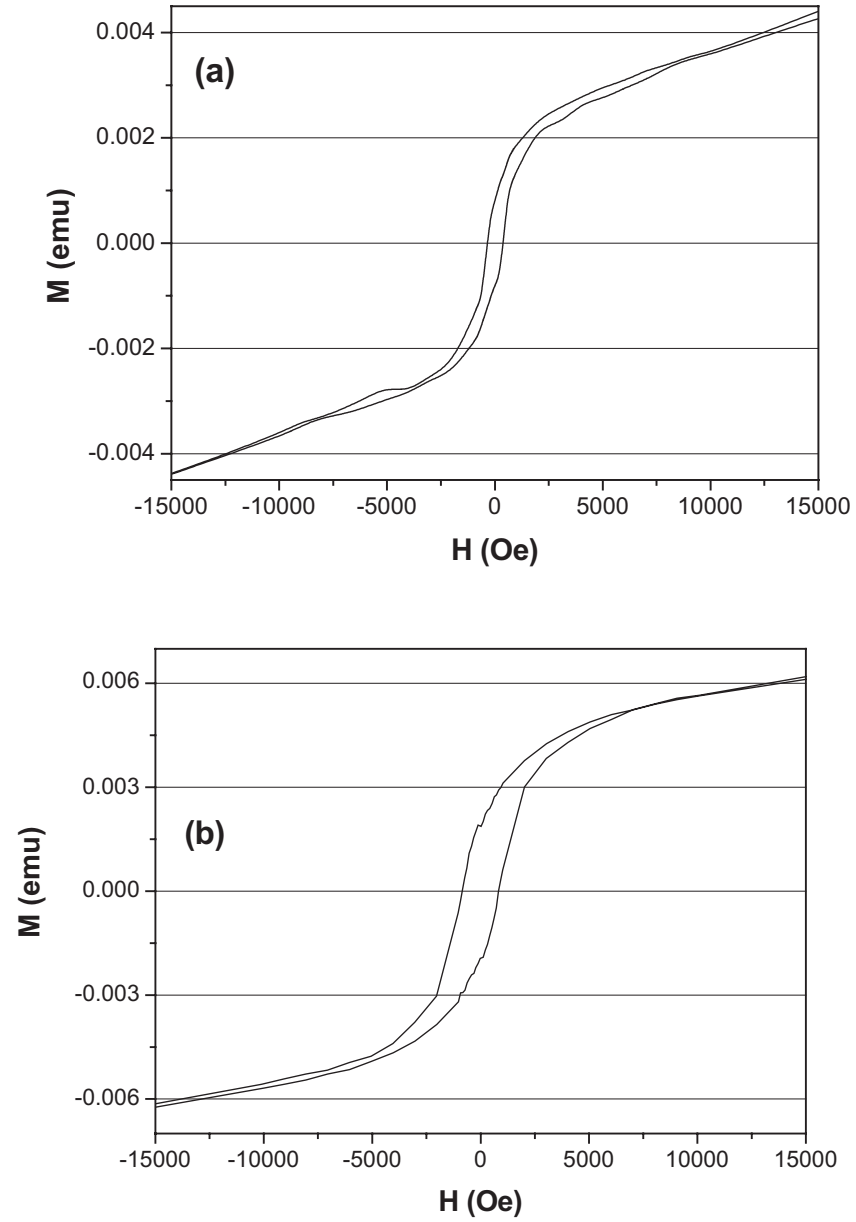

FIG. 5. Magnetization curves at $5 \mathrm{~K}$ of $\mathrm{Co}-\mathrm{SiO} 2 / \mathrm{AsGa}$ films with the applied field parallel to the film: (a) sample $1.1(x=80$ at. \%) and (b) sample $1.4(x=50$ at. $\%)$.

a)

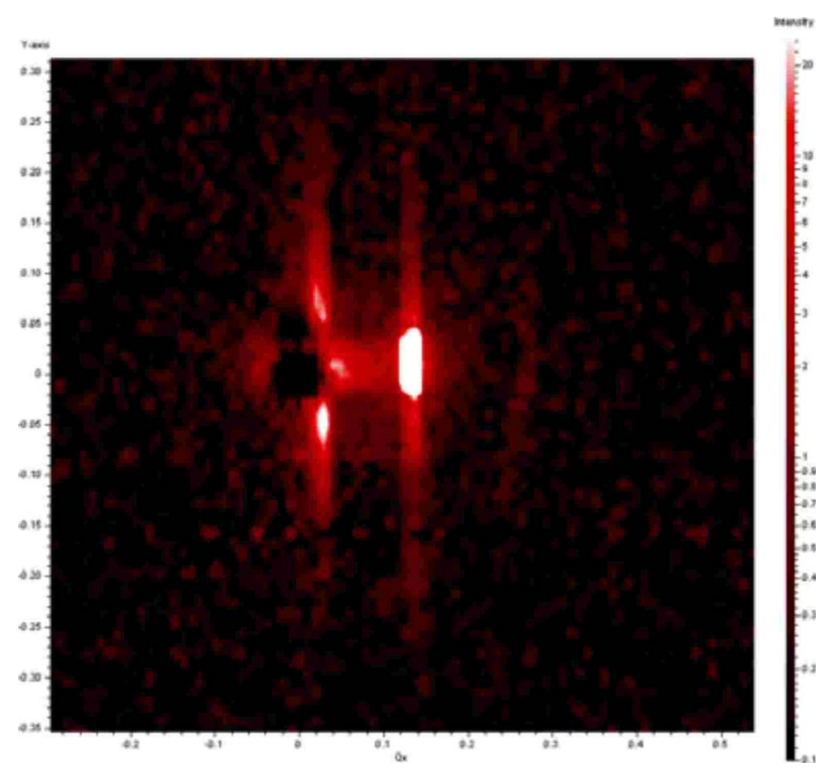

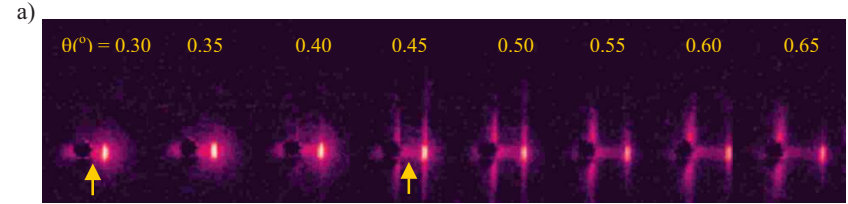

b)

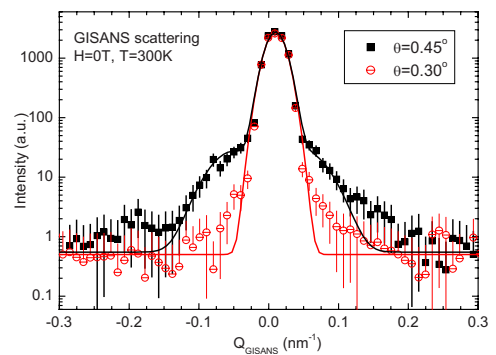

FIG. 7. (Color online) (a) GISANS signal of $\mathrm{Co} / \mathrm{SiO}_{2}$ as a function of incident angle $\theta$. Data were normalized by monitor counts. On both sides of the specular spot, GISANS scattering is seen to be maximum in intensity and width just above the critical angle $\theta_{c}=0.35^{\circ}-0.4^{\circ}$. Far above $\theta_{c}$, the GISANS signal rapidly fades away. (b) GISANS signal along the offspecular line (shown by arrows in Fig. 2) at angle $\theta=0.45^{\circ}$ (squares) and $\theta=0.30^{\circ}$ (circles). Continuous lines are Gaussian fits to the data as explained in the text.

mum frequency band of SWs in the DE geometry, we have chosen $H$ equal to $3000 \mathrm{Oe}$, which is the lowest possible value under the condition that the sample should be saturated. In usual continuous ferromagnetic films there are two contributions to the BLS spectrum. These are SWR, i.e., standing wave modes localized across the film thickness, and propagating magnetostatic DE modes. In our case the films are thick in which case the SWRs must be closely packed in the frequency space, thus superimposing and forming a wide peak of a characteristic shape. The Stokes and anti-Stokes

b)

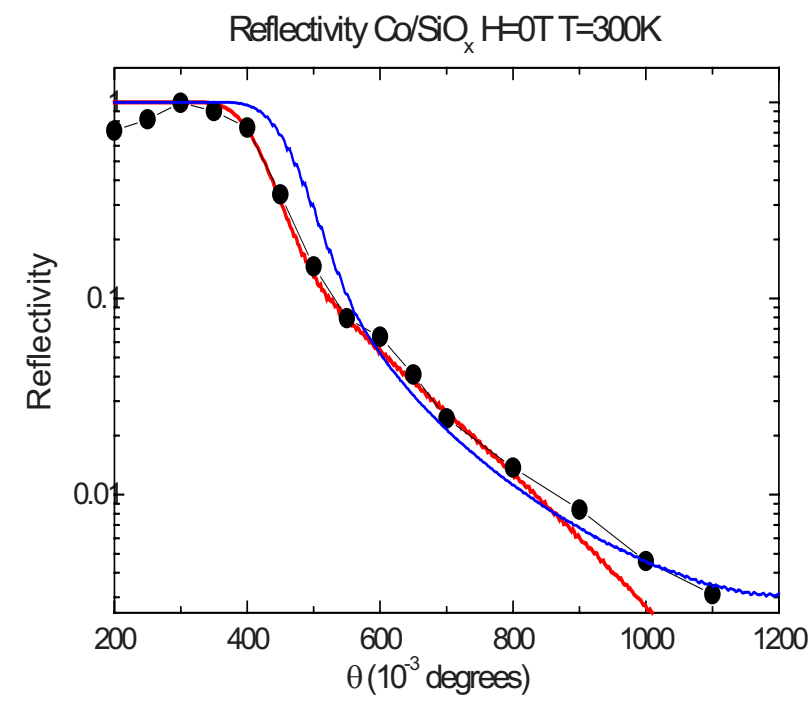

FIG. 6. (Color online) (a) 2D pattern of $\mathrm{Co} / \mathrm{SiO} 2$ obtained with an incident angle $\theta=0.5^{\circ}$. The direct beam is hidden by a neutron absorbing cadmium disk. The transmitted beam denotes the propagation through the sample. The specular spot $\left(\theta_{i}=\theta_{f}\right)$ and the surface diffraction scattering arise at the horizontal position $\left(Q_{z}\right)$ determined by the incident angle. (b) Neutron reflectivity curve of $\mathrm{Co} / \mathrm{SiO} 2$ obtained at zero field. The critical angle for total reflection is $0.35^{\circ}-0.4^{\circ}$. The continuous lines are fitted to the data as explained in the text. 
a)

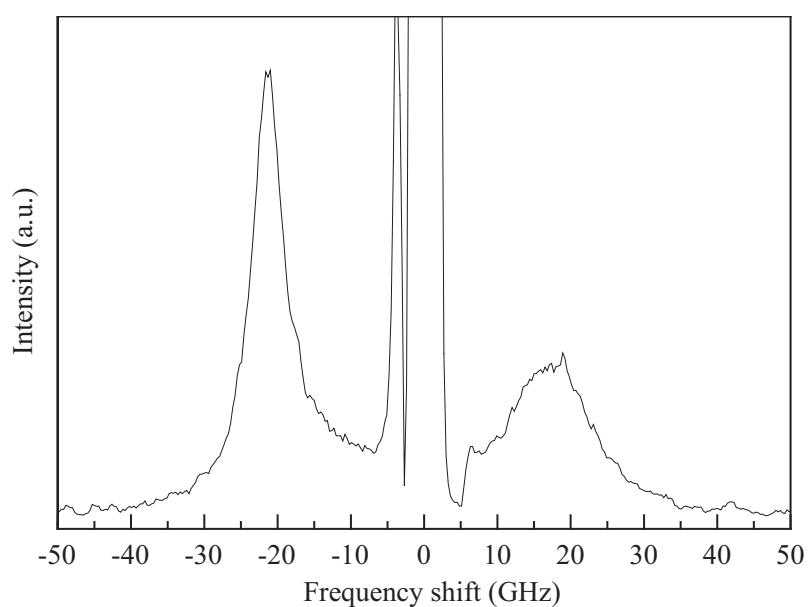

b)
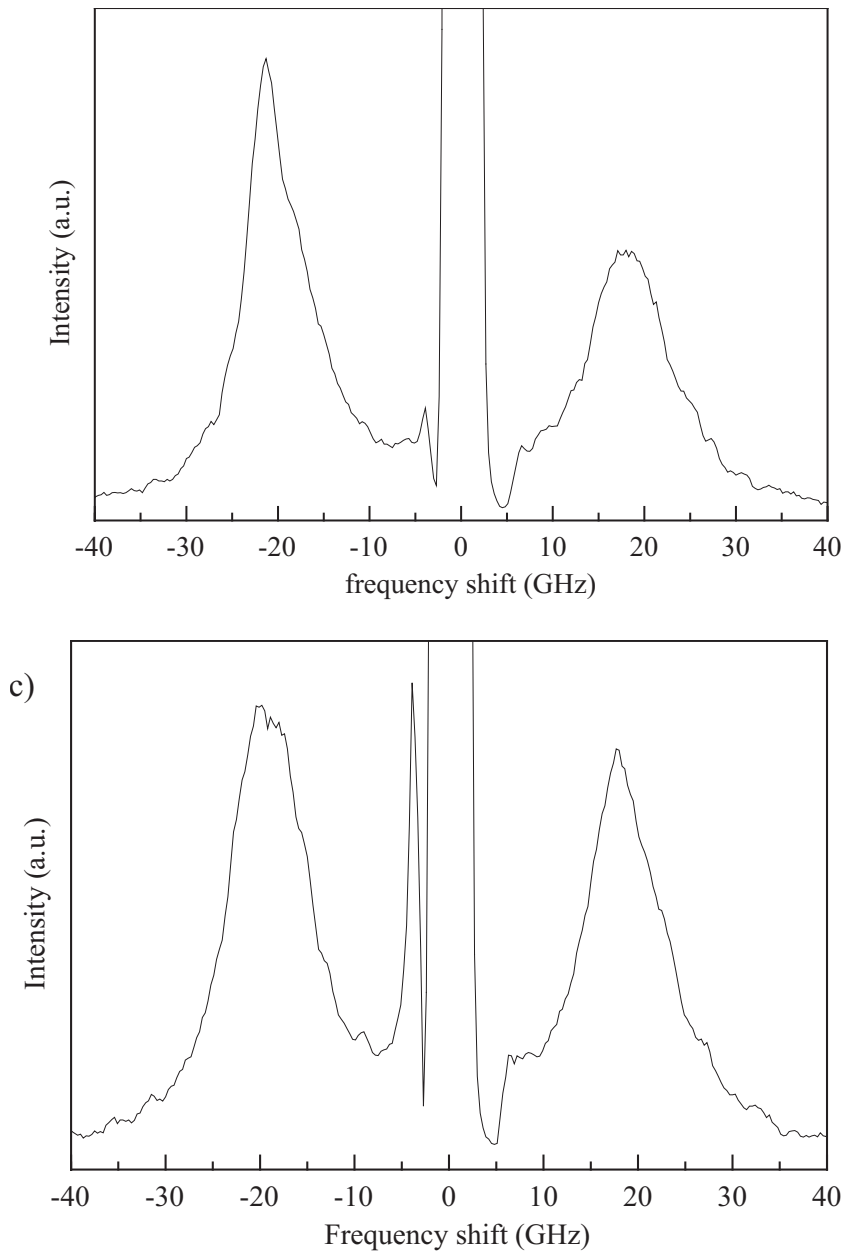

Fig.8.

FIG. 8. Typical BLS spectra measured at different angles of incidence (a) $\theta=50^{\circ}$, (b) $\theta=20^{\circ}$, and (c) $\theta=7^{\circ}$ on sample $1.1(x=80 \%)$. The peaks with negative frequency shifts are anti-Stokes ones and the peaks with positive shifts are Stokes ones.

responses (corresponding to the positive and the negative BLS frequency shifts, respectively) from SWRs are symmetric, while, in the DE geometry, the magnetostatic mode produces an asymmetric Stokes/anti-Stokes pattern. The major features of the typical spectra measured at different angles of incidence $\theta=50^{\circ}, \theta=20^{\circ}$, and $\theta=7^{\circ}$, given in Fig. 8 agree with these qualitative theoretical predictions. They are marked by a clearly observable asymmetry of Stokes/antiStokes lines that increases with the growth of the angle of incidence. The relative intensity of this DE peak grows considerably with the angle of incidence.

Figure 9 shows the experimentally measured dispersion relation $\omega(k)$ : frequency shifts of Stokes (triangles) and antiStokes peaks (circles and squares). Theoretical curves were plotted according to the classic DE formula. ${ }^{30}$

$$
\omega^{2}=\omega_{H}\left(\omega_{H}+\omega_{M}\right)+\frac{\omega_{M}^{2}}{4}[1-\exp (-2 k h)]
$$

where $\omega_{H}=\gamma H$ and $\omega_{M}=\gamma 4 \pi M_{s}$. Here $k$ is the SW wave number that is related to the angle of incidence $\theta$ by Eq. (1), $h$ is the film thickness, and $\gamma$ is the gyromagnetic ratio. We have investigated the SW dispersion, above all, as a function of the Co concentration $x$; Figs. 9(a)-9(d) correspond, respectively, to sample 1.1 ( $x=80$ at. \%) with $H=3000$ Oe, to sample $1.1(x=80$ at. $\%)$ with $H=5000$ Oe, sample $1.3(x$ $=60$ at. $\%)$ with $H=3000$ Oe, and sample1.4 (x $=50$ at. \%) with $H=3000$ Oe. In each figure both frequency shifts, Stokes and anti-Stokes, are plotted as a function of the angle of incidence.

In Fig. 9(a) the experimentally measured anti-Stokes frequency shift is denoted by squares and the Stokes shift is denoted by triangles. Circles correspond to the presence of a fine structure in the anti-Stokes line. The behavior of the anti-Stokes response, as reproduced by squares, is basically consistent with that of the theoretical dispersion of the DE mode given by a dashed curve. The following input fitting parameters were inserted in Eq. (2): applied field $H$ $=3000 \mathrm{Oe}$, saturation magnetization $4 \pi M_{s}=9300 \mathrm{G} \quad$ (a value consistent with the magnetization curve in the perpendicular film in Fig. 4), film thickness $h=600 \mathrm{~mm}$, and gyromagnetic factor $\gamma=2.82 \mathrm{GHz} / \mathrm{kOe}$.

However, there is an appreciable disagreement in the slope of the dispersion in theory and in experiment. This can be explained in the following way. For low values of angles of incidence, corresponding to small wave numbers $k h<1$, the asymmetric Stokes/Anti-Stokes contribution of DE mode to the overall optical response with respect to the symmetric broad peaks is small. The frequency of the DE mode, contributing mainly to the anti-Stokes line, displaces upward with $\theta$, according to Eq. (2). At the same time, symmetric non-DE peaks demonstrate a tendency to a slight shift downward, which is typical of backward volume SWs, which are characterized, in the presence of magnetic anisotropy, by a negative dispersion. However, in the DE approximation the spectrum of volume waves is infinitely degenerated, being described by a single horizontal line at the frequency $\omega_{H}\left(\omega_{H}+\omega_{M}\right)$ with no dispersion. This degeneracy can be removed by the exchange interaction, which splits this line into an infinite set of nonequidistant nonpropagating SWRs that take place across the film thickness. The degeneracy is also removed in the presence of magnetic anisotropy leading to propagating bulk modes (see, e.g., Ref. 31). In our case the presence of both factors is equally possible. For some particular types of anisotropy, e.g., for the uniaxial easy-axis anisotropy with the axis perpendicular to the film plane, the propagating bulk modes have a slight negative dispersion. 

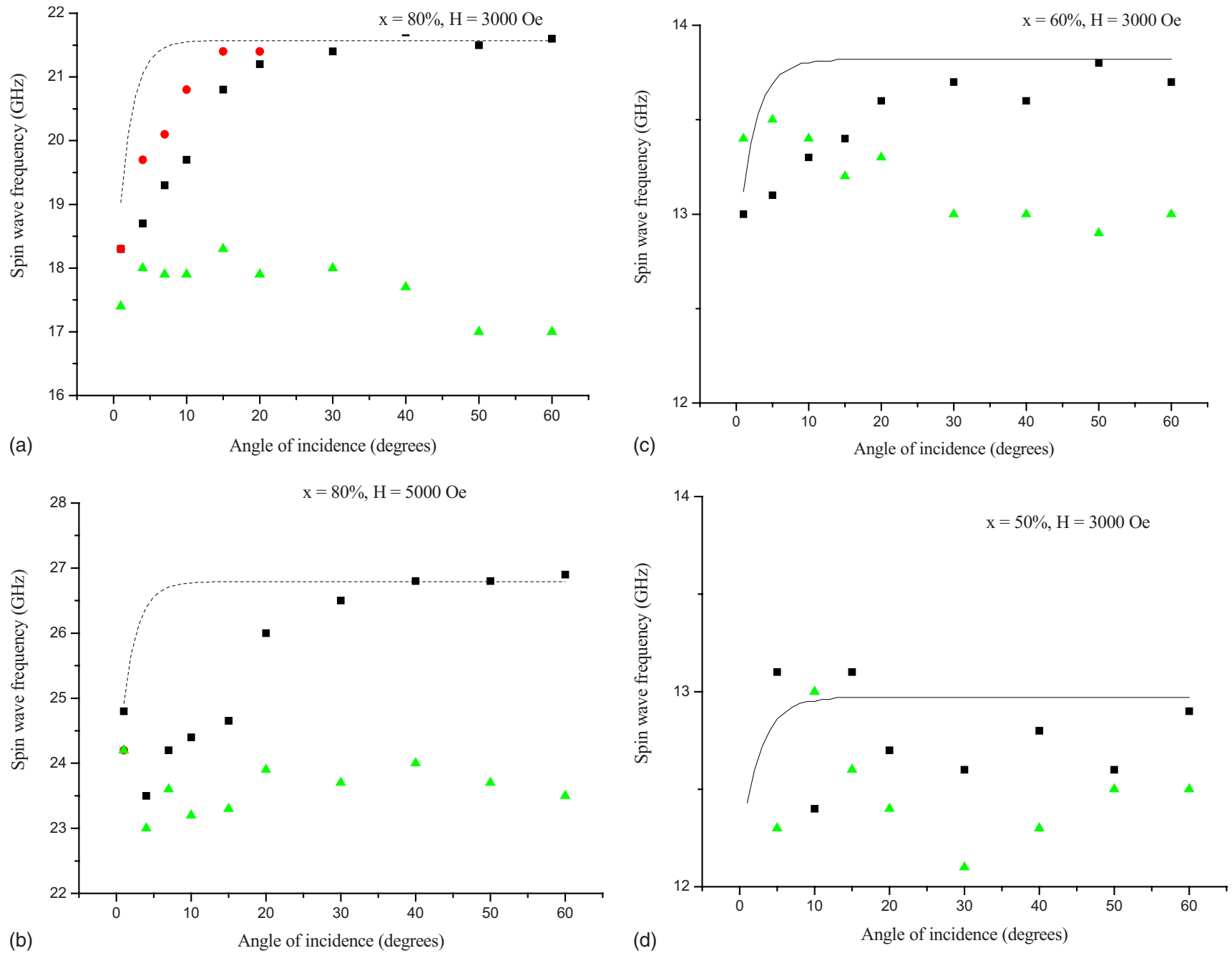

FIG. 9. (Color online) SW frequency vs angle of incidence. (a) Sample $1.1(x=80$ at. $\%$ ) with $H=3000$ Oe. (b) Sample 1.1 ( $x=80$ at. $\%)$ with $H$ $=5000$ Oe. (c) Sample 1.3 ( $x=60$ at. \%) with $H=3000$ Oe. (d) Sample $1.4(x=50$ at. \%) with $H=3000$ Oe.

Importantly, parallel to giving rise to the negative dispersion of the bulk modes, this anisotropy decreases the slope of the DE mode dispersion. Thus a superposition of two contributions, due to the DE mode and SWR, leads to a slower growth of frequency in the anti-Stokes line than that predicted by Eq. (2). It should also be noted that the smoothening of the slope of the dispersion curve at small angles of incidence is inevitable due to wide angle collection of the scattered light.

It is clearly seen in Fig. 9(a) that the DE dispersion slope is much better reproduced with the circles, taking into account the fine structure of the anti-Stokes line, than with the squares. This can be easily explained. Squares indicate the position of the overall anti-Stokes peak. Superposition of two major contributions, from DE and SWR modes, leads to a characteristic "two-level" anti-Stokes line structure reproduced experimentally. This is particularly well illustrated by Fig. 8(b). The contribution of the full set of bulk modes, creating the bottom level symmetrically reproduced in both Stokes and anti-Stokes lines, is very significant. Its presence pushes the overall anti-Stokes peak to lower frequencies characteristic of bulk modes. At the same time, it is clearly seen that the top subpeak on the second level of the anti-
Stokes line, representing the DE surface mode, shifts upwards with $K_{\mathrm{SW}}$ following the theoretical curve far more closely. Its pronounced Stokes/anti-Stokes asymmetry and frequency evolution confirm its DE origin. For higher values of angles of incidence it is the DE optical response that becomes predominant in the anti-Stokes peak, and the circles and the squares coincide. This can be regarded as another proof of the correctness of the hypothesis identifying the small subpeak with the DE surface mode.

Interestingly, for small angles of incidence, there can appear an extra-fine structure in the BLS spectral lines (see Fig. 10) in which case two mini peaks, representing DE and SWR contributions, can be resolved separately. In Fig. 10 the position of the "new-born" DE mini peak is marked with an arrow. This makes it possible, in low-angle BLS measurements, to separately trace the shift of a small peak indicating more precisely the frequency of the DE component in the anti-Stokes line, given by circles in Fig. 9(a). Its origin, as it is explained above, is due to different types of Stokes/antiStokes symmetry for DE and SWR modes and their superposition. Analysis of the extra-fine structure of BLS lines allows considerable improvement on the reproducibility of the dispersion slope in experiment, which corroborates the 
a)

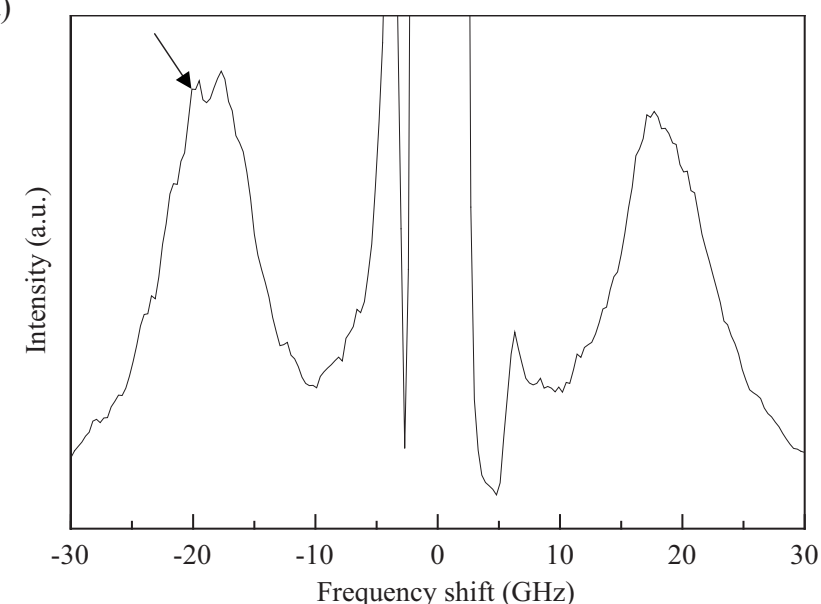

b)
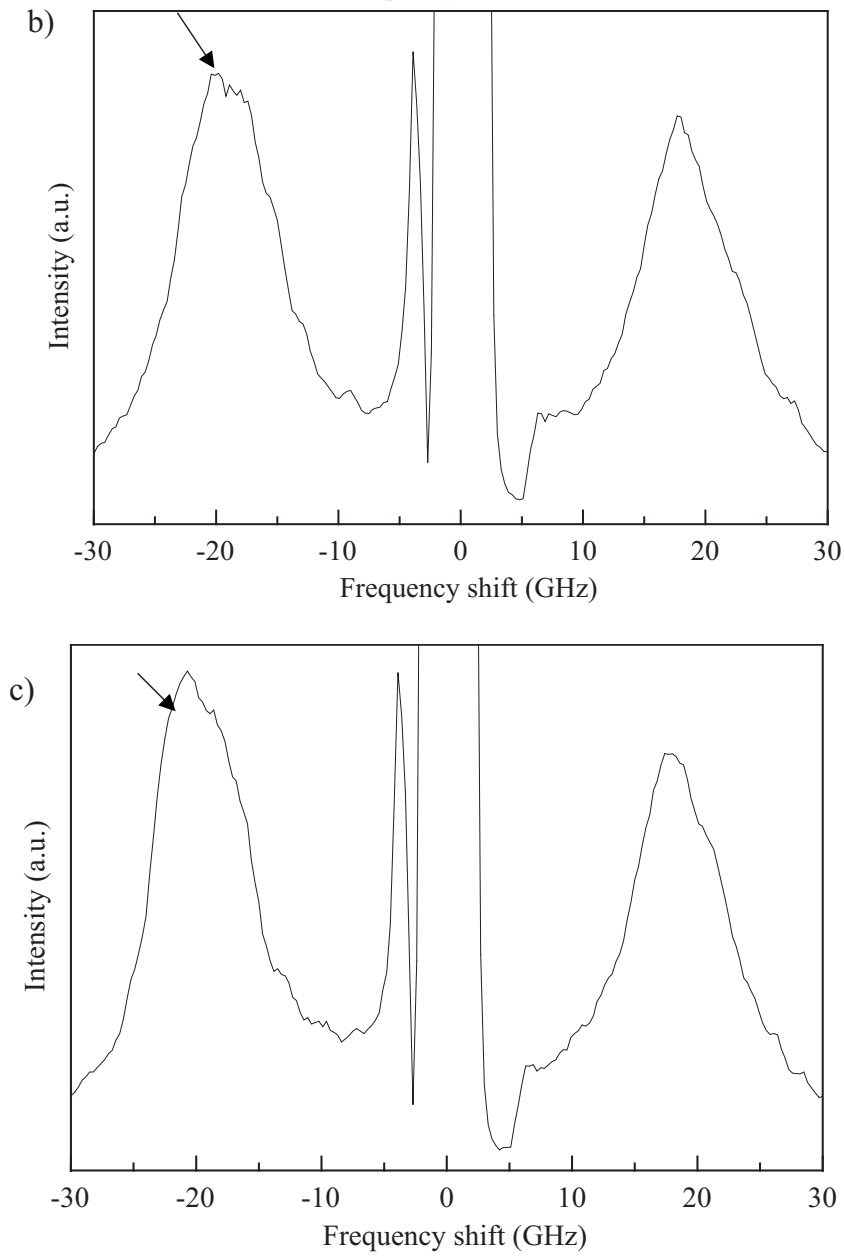

FIG. 10. Fine structure of BLS spectra. (a) $\theta=4^{\circ}$. (b) $\theta=7^{\circ}$. (c) $\theta=10^{\circ}$. The position of the mini peak, corresponding the "new-born" DE mode is marked with an arrow.

qualitative physical explanation outlined above. However, numerical simulations show that the "mini peaks" are not distinctly resolved in the BLS spectra, unless a very specific combination of major parameters, such as the effective exchange constant $A_{\text {eff }}$, saturation magnetization $4 \pi M_{s}$, film thickness $h$, and the external saturating magnetic field $H$, takes place. For example, there is no extra-fine structure observed for $H=5000$ Oe. It is not surprising: the frequency band of DE waves drops with the increase in $H$. This means that the DE dispersion becomes less pronounced and the fre- quency shift between DE and SWR responses decreases, becoming insufficient for separate resolution of the two mini peaks.

Figure 9(b) represents the DE dispersion for the case $H=5000$ Oe. It demonstrates features similar to those present in Fig. 9(a). The upward frequency shift of the antiStokes line, due to the DE MSW, is retained in Fig. 9(b). However, the DE and non-DE contributions are mixed to a higher extent, which leads to a less pronounced dispersion than for the previous case $H=3000 \mathrm{Oe}$. This is explained by the above-mentioned absence of the extra-fine structure pronounced for small $\theta$. To fit the experimental results, we put the value of the saturation magnetization equal to $9000 \mathrm{G}$. The behavior of the Stokes peak formed due to the scattering by non-DE modes is irregular, its frequency fluctuating in the vicinity of $\omega_{p}=\sqrt{\omega_{H}\left(\omega_{H}+\omega_{M}\right)}$.

Figure 9(c) presents the results of the measurements carried out on a less concentrated sample $1.3(x=60$ at. \%), 500 nm thick, with $H=3000$ Oe. For the theoretical curve, given by the solid line, we used a surprisingly low value of the saturation magnetization $4 \pi M_{s}=3800 \mathrm{G}$ (see Table I) as an input parameter. Apparently, the field of 3000 Oe is not sufficient to fully saturate the sample, which attests to the SPM state of the film. As in the case of the previous sample 1.1 with a higher concentration, a branch with positive dispersion in the anti-Stokes line (squares) and another branch with negative dispersion in the Stokes line (triangles) are clearly observed. However, in contrast to the previous case the values of SW frequencies at low angles of incidence are higher in the Stokes line.

Finally, in Fig. 9(d), illustrated are the results obtained on sample 1.4 with the lowest Co concentration. No proof of dispersion has been found either in the Stokes or anti-Stokes line. It should be noted, however, that the frequency band of the DE mode, calculated for $4 \pi M_{s}=3200 \mathrm{G}$ as an input parameter, is as narrow as $0.55 \mathrm{GHz}$, while the BLS peaks are extremely wide $(\Delta H \approx 10 \mathrm{GHz})$, which makes detection of dispersion very difficult. At the same time, the mean frequency shift in the anti-Stokes line is definitely larger than in the Stokes one.

The asymmetry of the Stokes/anti-Stokes spectra, being a major feature of a collective DE mode, is interesting to study as a function of the Co concentration. Such results are presented in Fig. 11. They were obtained from the above mentioned three samples for the same angle of incidence $\theta$ $=20^{\circ}$. The degree of the asymmetry drops with decreasing Co concentration. It can be seen that the two spectra, corresponding to low concentrations, are more symmetric and the DE and SWR contributions are practically inseparable. According to our measurements of the surface resistance, the percolation threshold lies in the vicinity of $x=60$. This agrees well with the figures reported in a similar structure in Ref. 8: $x \approx 65$. In other words, these samples are in the SPM state, in which case there is no more exchange. This means that the spectrum of volume waves becomes degenerated or, in the presence of magnetic anisotropy, almost degenerated. This leads to a very high density of states corresponding to volume waves, which appreciably increases their contribution to the BLS spectrum, thus making its structure much more 

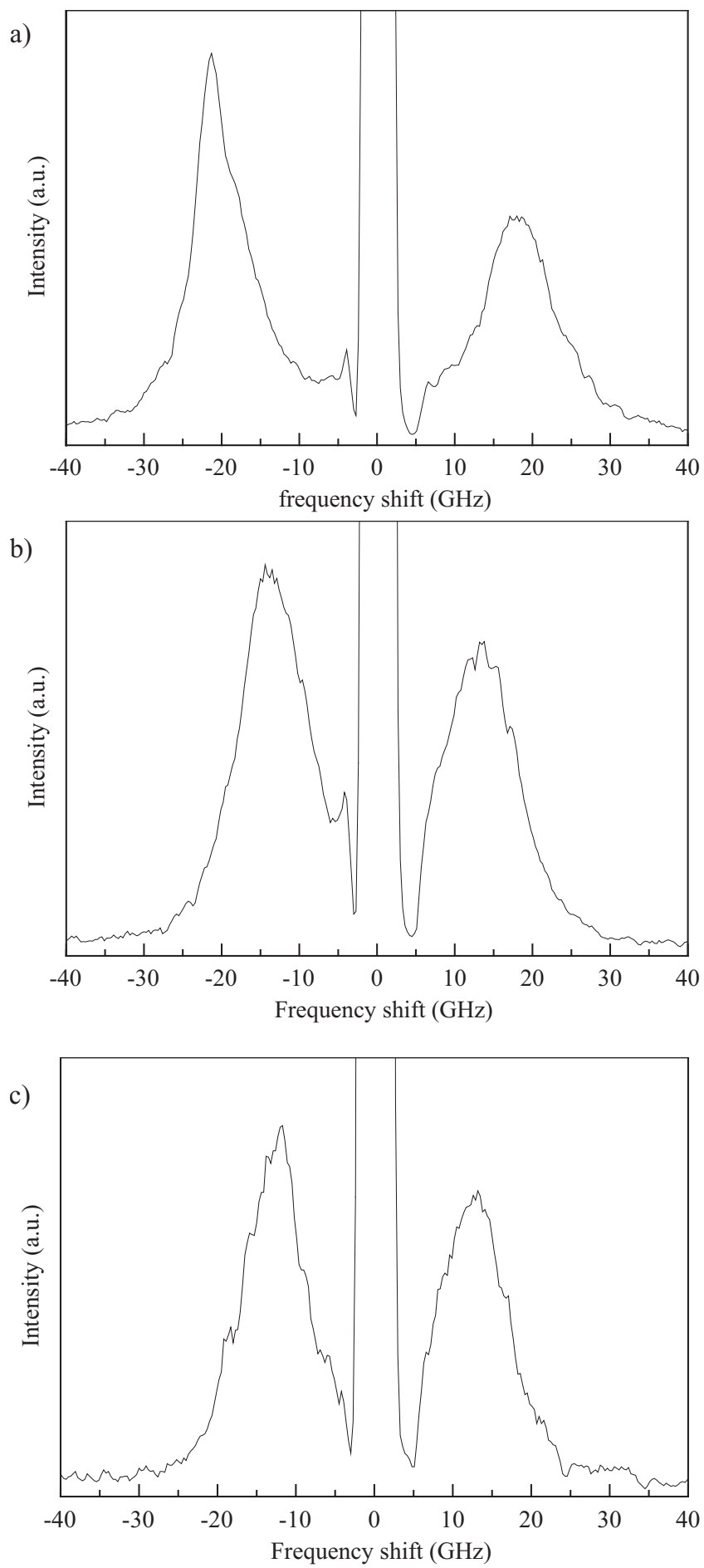

FIG. 11. Asymmetry of Stokes/anti-Stokes lines in BLS spectra for a fixed angle of incidence $\theta=20^{\circ}$ and magnetic field $H=3000$ Oe for different Co concentrations. (a) Sample $1.1 \quad(x=80$ at. \%). (b) Sample $1.3 \quad(x$ $=60$ at. $\%)$. (c) Sample $1.4(x=50$ at. $\%)$.

symmetric. Another consideration to be taken into account is the variation in the index of refraction. With decreasing Co concentration the films become more transparent and the Stokes/anti-Stokes pattern appears more symmetric.

To complete the characterization of samples we have measured the dependences of the SW frequency on the external magnetic field $H$. The results of the measurements carried out on the same three samples at the angle $\theta=20^{\circ}$ in the magnetic field decreasing from $10 \mathrm{kOe}$ down to $0.5 \mathrm{kOe}$

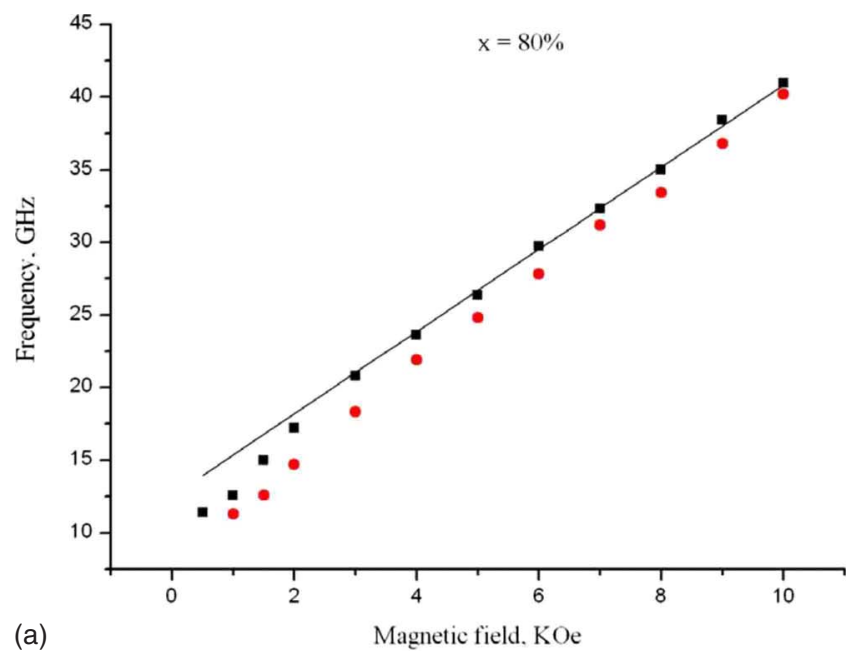

(a)
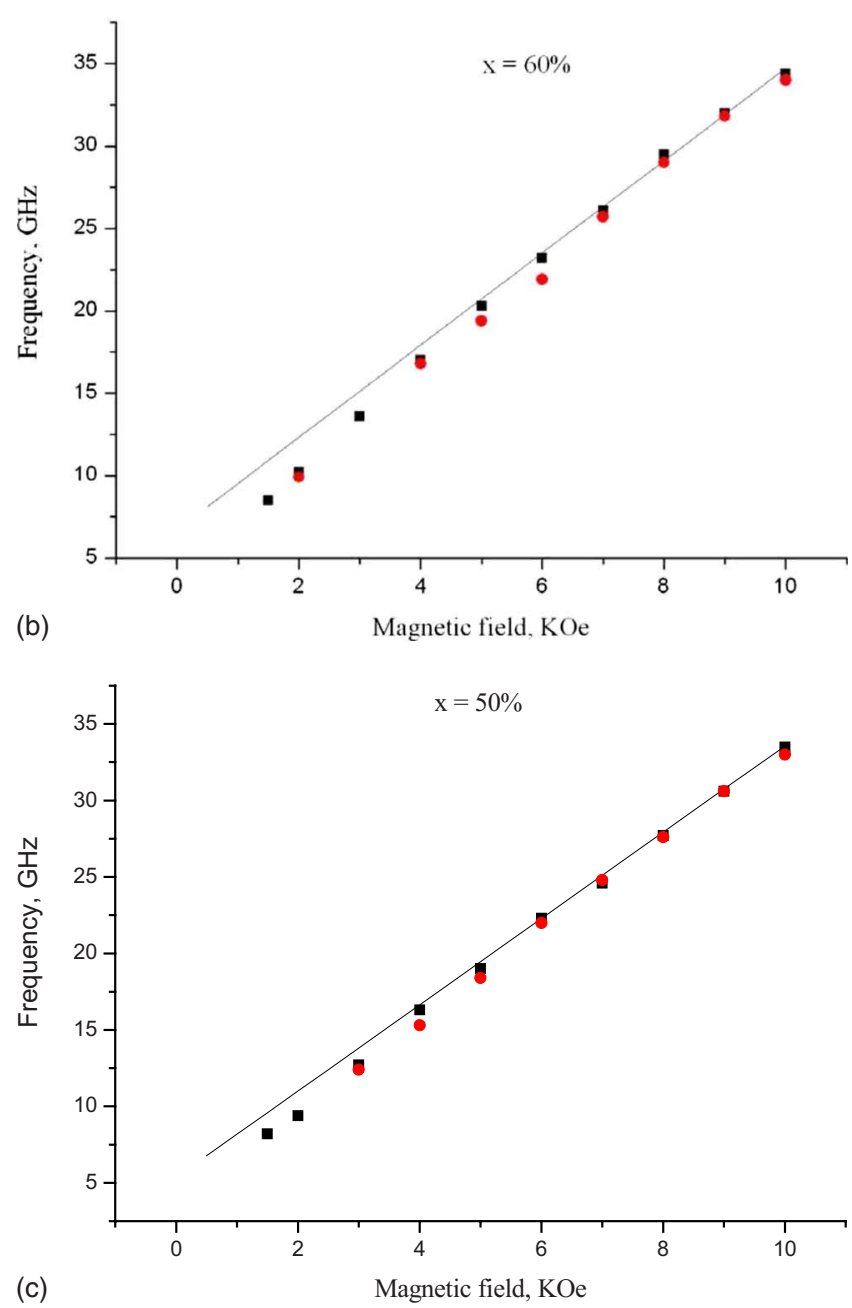

FIG. 12. (Color online) SW frequency vs magnetic field in (a) sample 1.1 $(x=80$ at. $\%)$, (b) sample $1.3(x=60$ at. $\%)$, and (d) sample $1.4(x$ $=50$ at. $\%$ ). The angle of incidence $\theta$ is equal to $20^{\circ}$.

are given in Fig. 12. In all three cases there is a tendency for saturation to begin from $H=3 \mathrm{kOe}$ in the most concentrated film and from 4 to $5 \mathrm{kOe}$ for the other two. The meaning of the symbols is as before (see, e.g., Figure 9): the squares correspond to the frequency of the overall anti-Stokes BLS line and the circles to that of the purely DE subpeak in the same line. For $\theta=20^{\circ}$ this frequency difference is non- 
negligible, especially in the concentrates sample. With growth of $H$ the $\mathrm{DE}$ and bulk spectra come closer, hence this frequency splitting visibly decreases. Not surprisingly, in diluted SPM samples with less pronounced dispersion, the splitting is considerably smaller.

In conclusion it should be noted that the relative shapes of the two BLS spectral lines, the Stokes and anti-Stokes, carry very important information on the nature of the magnetic excitations existing in a ferromagnetic film. Since the BLS is an optical technique, knowledge of the optical properties of the samples is essential for adequate interpretation of the experimental results. For example, the depth of penetration of the light wave in a film, dependent of the complex index of refraction, influences considerably one of the major features of a BLS spectrum: the degree of Stokes/anti-Stokes asymmetry.

The index of refraction, or in the case of a nanocomposite material effective dielectric constant of the samples, can be found from one of the mixture equations in the framework of the effective medium theory. For the composites with magnetic conductive inclusions, the Bruggeman equation is one of the most appropriate approaches. ${ }^{32}$ For the nanoparticles of spherical shape it is written as follows: ${ }^{33}$

$$
f \frac{\varepsilon_{2}-\varepsilon_{\mathrm{eff}}}{\varepsilon_{2}+2 \varepsilon_{\mathrm{eff}}}+(1-f) \frac{\varepsilon_{1}-\varepsilon_{\mathrm{eff}}}{\varepsilon_{1}+2 \varepsilon_{\mathrm{eff}}}=0
$$

where $\varepsilon_{1}$ is a dielectric constant of the matrix, $\varepsilon_{2}$ is a dielectric constant of the inclusion, and $f$ is a volumetric filling factor. For the chosen samples the Bruggeman equation gives the following values for the effective dielectric constants: $\varepsilon_{\text {eff }}=0.3+i 6.3$ (sample 1.1), $\varepsilon_{\text {eff }}=3.0+i 3.3$ (sample 1.3), and $\varepsilon_{\text {eff }}=3.2+i 2.2$ (sample 1.4). To calculate the bulk dielectric constants for the silica matrix and Co nanoparticles we used the values given in Ref. 34 .

We quantified the value of the exchange constant $A$ by comparing the measured and the theoretical shapes of the Stokes/anti-Stokes BLS lines. ${ }^{35,36}$ For our computer simulations, considering the thermal stochastic nature of the magnons, we employed the code based on the FDT (see, for example, Ref. 37) and optical Green's functions for a multilayer, described in more detail in Ref. 38.

The intensity of the light scattered at a given angle by thermal magnons is proportional to the Fourier transform of the correlation function $\left\langle m_{i}\left(x^{\prime}, \vec{r}, t\right) m_{j}(x, \vec{r}=0, t=0)\right\rangle$. Applied to a ferromagnetic system, the FDT relates these correlation functions to the dynamic susceptibilities $g_{i j}\left(x, x^{\prime}, \vec{K}, \omega \pm i \varepsilon\right)$,

$$
\begin{aligned}
\int d t d^{2} r\left\langle m_{i}\left(x^{\prime}, \vec{r}, t\right) m_{j}(x, \vec{r}=\right. & 0, t=0)\rangle \exp [i(-\vec{K} \cdot \vec{r}+\omega t)] \\
\propto & {\left[g_{i j}\left(x, x^{\prime}, \vec{K}, \omega+i \varepsilon\right)\right.} \\
& \left.-g_{i j}\left(x, x^{\prime}, \vec{K}, \omega-i \varepsilon\right)\right],
\end{aligned}
$$

in the limit $\varepsilon \rightarrow 0 . g_{i j}\left(x, x^{\prime}, \vec{K}, \omega \pm i \varepsilon\right)$, being responses to delta-type excitations $h_{i}(x)=\delta\left(x-x^{\prime}\right)$, can be as well regarded as magnetic Green's functions. Optical properties of the highly absorbing metal multilayer structures are taken into account via the optical Green's functions formalism. The
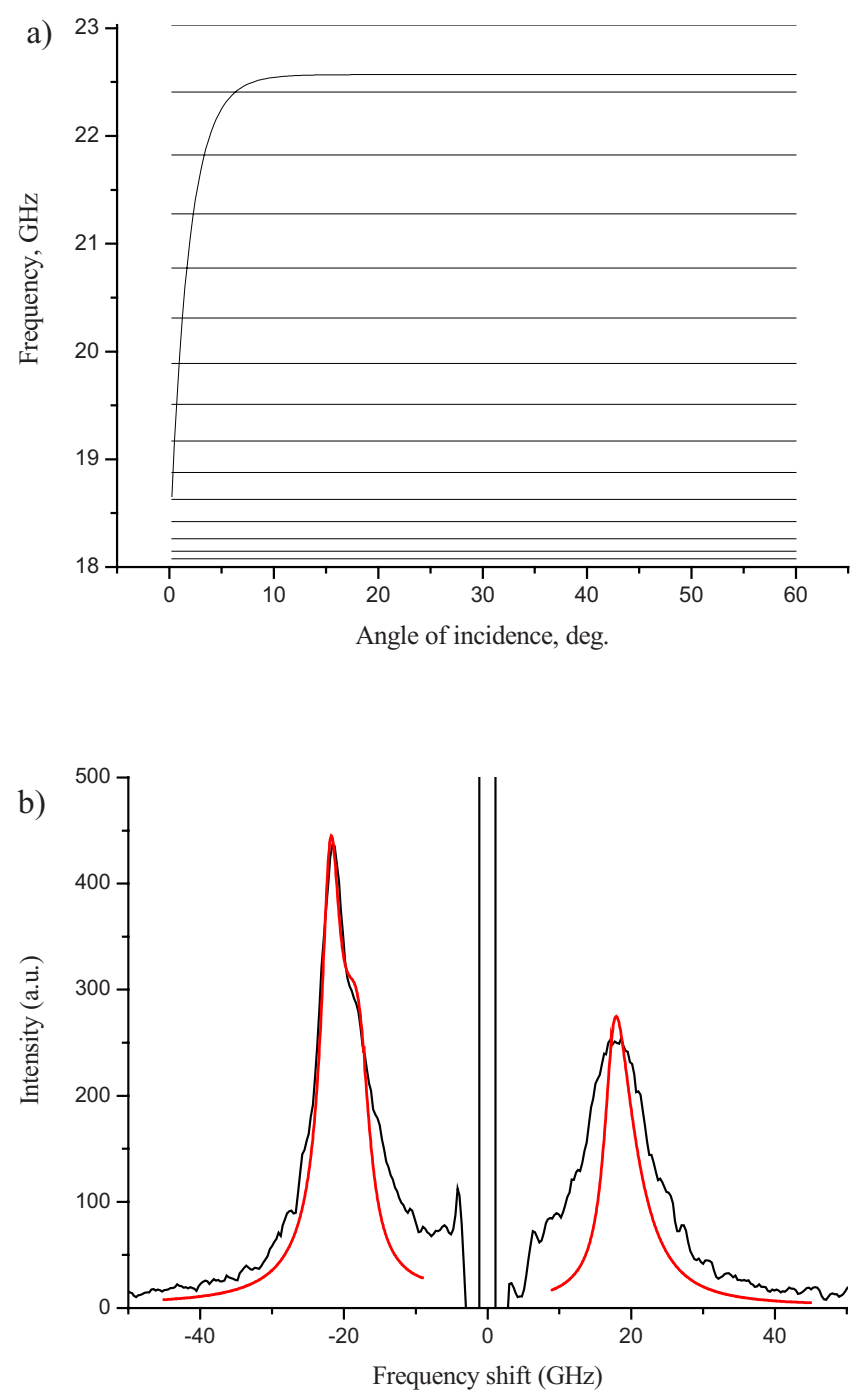

FIG. 13. (Color online) (a) SW dispersion and (b) computer simulations of the BLS line structure in the thick sample 1.1. The external parameters are the same as in Fig. 14: $H=3000$ Oe and $\theta=20^{\circ}$. The best fit for the effective exchange constant has a somewhat greater value $A_{\mathrm{eff}}=0.8 \times 10^{-6} \mathrm{erg} \mathrm{cm}^{-1}$.

principles of this powerful theoretical approach have been developed in the late $70 \mathrm{~s} .{ }^{39-41}$

Some results of such numerical modeling are given in Fig. 13. Figure 13(a) illustrates the density of SWR states in film corresponding to sample 1.1: 14 SWR modes are localized within the frequency span of existence of the DE mode. Calculations were made for the following conditions: $H$ was equal to 3000 Oe and $A_{\text {eff }}$ was taken equal to its best fit value $A_{\text {eff }}=0.8 \times 10^{-6} \mathrm{erg} \mathrm{cm}^{-1}$, which had been obtained from the numerical simulations of the fine structure of the BLS spectra [see Fig. 13(b)]. To find the best fit value of $A_{\text {eff }}$ we used as the main criterion numerical reproduction the characteristic two-level structure of the anti-Stokes line. What is very important, is that the height of the step between the upper and lower levels strongly depends on the adopted $A_{\text {eff }}$ value. This provides for reliable fitting of the effective exchange constant, which has turned out to be appreciably smaller than in the bulk Cobalt samples, ${ }^{42} A_{\text {eff }}=1.8 \times 10^{-6} \mathrm{erg} \mathrm{cm}^{-1}$. The value of the width of the FMR line was taken equal to 500 Oe.

The reduced value of $A_{\text {eff }}$ can be explained, qualitatively, 


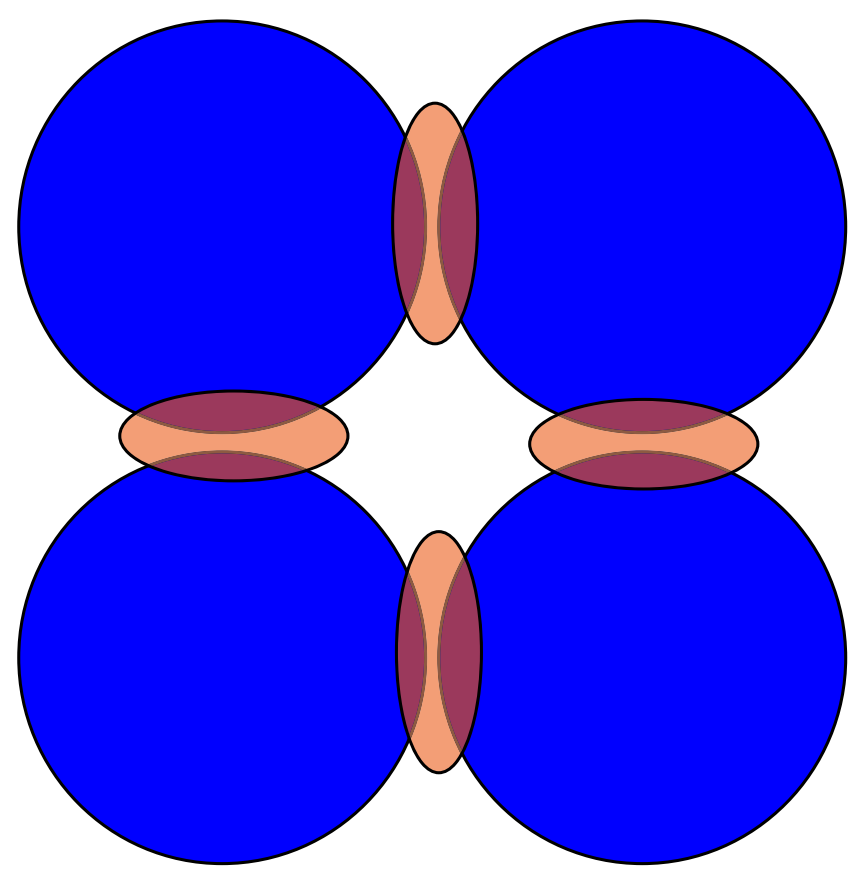

FIG. 14. (Color online) Schematic illustration of the localized point character of interparticle exchange interaction.

by the geometry of the nanocomposite structure (see Fig. 14). As an example, let us consider a system of four closely spaced particles. Although the theory of the interlayer exchange coupling between two ferromagnetic films via tunneling through a dielectric coupling has been developed simultaneously with that for more common metallic spacers, ${ }^{43,44}$ there is much less experimental evidence confirming theoretical predictions. However, one can conclude that effective coupling can be achieved only when the dielectric spacer thickness is less than $1 \mathrm{~nm} .^{45,46}$ The order of smallness of this figure, for a nanocomposite medium with Co particles of spherical symmetry, is corroborated in Refs. 14 and 17. In these papers effects directly linked to interparticle tunneling of electrons have been investigated.

Even if the particles touch one another, the zones of effective tunneling (see Fig. 14) do not cover all the "active" particle surface. Thus, in the case of noncubic nanoparticles the exchange constant $A_{\text {eff }}$, even in the limiting case of zero interparticle spacing, will never reach its bulk value. The value of $A_{\text {eff }}$ will drop off with the increase in the interparticle spacing. It will also decrease as a function of the particle size compared to the characteristic length of $1 \mathrm{~nm}$, since in this case the ratio of the actively interacting particle surface to its overall surface diminishes. Another important factor to be taken into account is the complex geometry, which can lead to even smaller values of $A_{\text {eff }}{ }^{47}$

It should be added that this point interparticle exchange contact will perturb, through the mixed Hoffman boundary conditions, ${ }^{48}$ the dynamic magnetization within each particle which becomes inhomogeneous. In other words the classic superspin approximation, regarding single domain particles as a magnetic point with no internal structure, is no longer sufficient to adequately describe the high-frequency dynamics of a SFM film. The effective exchange constant $A_{\text {eff }}$ of the nanogranular medium will depend on both $A$ (intraparticle constant) and $A_{12}$ (interparticle constant), as well as the particle size and particle spacing. It should be noted that the issue of the applicability of the superspin/macrospin model attracts more and more attention recently. ${ }^{49,50}$ In reality, the exchange building mechanisms are even more complicated, including formations of clusters in the composite materials with concentrations above the percolation threshold. ${ }^{51}$

Until now we are addressing the situation when the spatial period of a SWR is much greater than the particle size. If these two parameters are comparable, the physical nature of the magnetic excitations will change dramatically.In this case the SWRs will be localized on individual particles and coupled through interparticle exchange. The role of the DDIs will be negligible in this case.

The values of $H_{a}$ and $A_{\text {eff }}$ obtained from numerical simulations of SW dispersion and BLS line shape allow estimation of the minimum film thickness for existence of week stripe domains, ${ }^{52}$

$$
t_{0}=2 \pi \sqrt{\frac{A}{K}} \text { with } \quad K=\frac{M H_{a}}{2} .
$$

The negative dispersion clearly seen in Fig. 9(a) implies the presence of a magnetic uniaxial anisotropy of the order of hundreds of Oersteds, while, according to the simulations given in Fig. 13(b), $A_{\text {eff }}=0.8 \times 10^{-6} \mathrm{erg} \mathrm{cm}^{-1}$. The value of $t_{0}$ in our films according to the above formula lies in the range 100-150 $\mathrm{nm}$, which explains the existence of weak stripe domains in sample 1.1 .

Interestingly, similar domain structures have been observed on Cobalt cluster-assembled thin films. ${ }^{53}$ In this case, particles were piled with no matrix to fix their spatial position. They were supposed to be in direct mechanical and electric contact, which correspond, approximately, to the geometry of our concentrated samples $(x=80 \%)$. The value of $A_{\text {eff }}=0.23 \times 10^{-6} \mathrm{erg} \mathrm{cm}^{-1}$ reported in this work is less than the value estimated by us. This is in line with our qualitative theoretical model, exposed above: the effective size of the agglomerate-type particles in Ref. 53 was of the order of 10 $\mathrm{nm}$, i.e., at least two times greater than in our case.

\section{CONCLUSION}

In the present paper, the evolution of dynamic behavior of magnetic excitations in a nanocomposite $\mathrm{Co}_{x} \mathrm{SiO}_{21-x}$ ferromagnetic film during SPM-to-SFM transition has been investigated by means of the BLS technique in the frequency range from 10 to $50 \mathrm{GHz}$ with $\mathrm{SW}$ wave number varying from $3 \times 10^{3}$ to $2 \times 10^{5} \mathrm{~cm}^{-1}$. More specifically, the transformation of purely dipole magnetostatic modes into dipoleexchange SWs with the growths of Co concentration has been traced.

Not surprisingly, less concentrated samples $(x$ $<60$ at. \%) demonstrated SPM behavior, identical to that of a slightly undersaturated ferromagnetic film in the absence of exchange. It is characterized by a weakly pronounced SW dispersion and a reduced Stokes/anti-Stokes asymmetry. The latter is a consequence of a high density of states in the vicinity of $\omega_{p}=\sqrt{\omega_{H}\left(\omega_{H}+\omega_{M}\right)}$ due to the degeneracy of the Backward Volume Magnetostatic Wave (BVMSW) modes. 
With the growth of the sample concentration, the features typical of the presence of nonvanishing exchange interactions begin to manifest themselves. The BVMSW degeneracy is removed and the contributions of the DE and backward volume modes can be identified in the Stokes/antiStokes BLS spectral lines, which allows quantitative estimation of such film parameters such as the effective exchange constant and magnetic anisotropy. Statically, the ferromagnetic nature of the concentrated sample $(x=80$ at. \%) manifests itself via the existence of weak stripe domains, detected by MFM/AFM imaging and by GISANS.

\section{ACKNOWLEDGMENTS}

This work was supported by the French Ministry of National Education and Research (ACI Grant No. NR0095 "NANODYNE") and partly by the "Australian Research Council," RFBR (Grant No. 07-02-91588-ASP), and Russian foundation "Dynasty." G.W. and A.Z. gratefully acknowledge financial support from EPSRC (UK). The authors gratefully acknowledge the assistance of Dr. V. M. Lebedev (PNPI, Gatchina, Leningrad region, Russia) for determination of the film composition by Rutherford backscattering spectroscopy. We would like to thank Professor P. Moch for helpful discussions.

${ }^{1}$ J. Inoue and S. Maekawa, Phys. Rev. B 53, R11927 (1996)

${ }^{2}$ K. Yakushiji, S. Mitani, K. Takanashi, J.-G. Ha, and H. Fujimori, J. Magn. Magn. Mater. 212, 75 (2000).

${ }^{3}$ J. S. Micha, B. Dieny, J. R. Régnard, J. F. Jacquot, and J. Sort, J. Magn. Magn. Mater. 272-276, E967 (2004).

${ }^{4}$ S. Sankar, D. Dender, J. A. Borchers, D. J. Smith, R. W. Erwin, S. R. Kline, and A. E. Berkowits, J. Magn. Magn. Mater. 221, 1 (2000).

${ }^{5}$ L. Néel, Ann. Geophys. (C.N.R.S.) 5, 99 (1949).

${ }^{6}$ W. F. Brown, Jr., Phys. Rev. 130, 1677 (1963).

${ }^{7}$ J. Hesse, H. Bremers, O. Hupe, M. Veith, E. W. Fritscher, and K. Valtchev, J. Magn. Magn. Mater. 212, 153 (2000).

${ }^{8}$ M. Azeggagh and H. Kachkachi, Phys. Rev. B 75, 174410 (2007).

${ }^{9}$ C. Djurberg, P. Svedlindh, P. Nordblad, M. F. Hansen, F. Bødker, and S Mørup, Phys. Rev. Lett. 79, 5154 (1997).

${ }^{10}$ T. Jonsson, P. Nordblad, and P. Svedlindh, Phys. Rev. B 57, 497 (1998).

${ }^{11}$ P. E. Jönsson, S. Felton, P. Svedlindh, P. Nordblad, and M. F. Hansen, Phys. Rev. B 64, 212402 (2001).

${ }^{12}$ F. Luis, F. Petroff, J. M. Torres, L. M. García, J. Bartolomé, J. Carrey, and A. Vaurès, Phys. Rev. Lett. 88, 217205 (2002).

${ }^{13}$ J. Nogués, V. Skumryev, J. Sort, S. Stoyanov, and D. Givord, Phys. Rev. Lett. 97, 157203 (2006).

${ }^{14}$ L. F. Schelp, A. Fert, F. Fettar, P. Holody, S. F. Lee, J. L. Maurice, F. Petroff, and A. Vaurès, Phys. Rev. B 56, R5747 (1997).

${ }^{15}$ W. Kleemann, O. Petracic, Ch. Binek, G. N. Kakazei, Yu. G. Pogorelov, J. B. Sousa, S. Cardoso, and P. P. Freitas, Phys. Rev. B 63, 134423 (2001).

${ }^{16}$ X. Chen, S. Bedanta, O. Petracic, W. Kleemann, S. Sahoo, S. Cardoso, and P. P. Freitas, Phys. Rev. B 72, 214436 (2005).

${ }^{17}$ S. Bedanta, T. Eimüller, W. Kleemann, J. Rhensius, F. Stromberg, E. Amaladass, S. Cardoso, and P. P. Freitas, Phys. Rev. Lett. 98, 176601 (2007).

${ }^{18}$ A. Butera, J. N. Zhou, and J. A. Barnard, Phys. Rev. B 60, 12270 (1999).
${ }^{19}$ F. Yildiz, S. Kazan, B. Aktas, S. I. Tarapov, and B. Granovsky, J. Magn. Magn. Mater. 305, 24 (2006).

${ }^{20}$ J. Gómez, A. Butera, and J. A. Barnard, Phys. Rev. B 70, 054428 (2004).

${ }^{21}$ Y. Roussigné, S. M. Chérif, C. Dugautier, and P. Moch, Phys. Rev. B 63, 134429 (2001).

${ }^{22}$ S. O. Demokritov, B. Hillebrands, and A. N. Slavin, Phys. Rep. 348, 441 (2001).

${ }^{23}$ G. Gubbiotti, P. Candeloro, L. Businaro, E. Di Fabrizio, A. Gerardino, R. Ziveri, M. Conti, and G. Carlotti, J. Appl. Phys. 93, 7595 (2003).

${ }^{24}$ S. L. Vysotskii, S. A. Nikitov, and Yu. A. Filimonov, JETP 101, 547 (2005).

${ }^{25}$ M. P. Kostylev, A. A. Stashkevich, and N. A. Sergeeva, Phys. Rev. B 69, 064408 (2004).

${ }^{26}$ G. Gubbiotti, S. Tacchi, G. Carlotti, P. Vavassori, N. Singh, S. Goolaup, A. O. Adeyeye, A. Stashkevich, and M. Kostylev, Phys. Rev. B 72, 224413 (2005).

${ }^{27}$ A. I. Stognij, N. N. Novitskii, and O. M. Stukalov, Tech. Phys. Lett. 28, 17 (2002).

${ }^{28}$ A. I. Stognij, N. N. Novitskii, and O. M. Stukalov, Tech. Phys. Lett. 29, 147 (2003).

${ }^{29}$ SIMULREFLEC, package available at http://www-llb.cea.fr/prism/programs/ programs.html.

${ }^{30}$ R. W. Damon and J. R. Eshbach, J. Phys. Chem. Solids 19, 308 (1961).

${ }^{31}$ B. A. Kalinikos, N. V. Kozhus, M. P. Kostylev, and A. N. Slavin, J. Phys.: Condens. Matter 2, 9861 (1990).

${ }^{32}$ J. L. Menendez, B. Bescos, G. Armelles, R. Serna, J. Gonzalo, R. Doole, A. K. Petford-Long, and M. I. Alonso, Phys. Rev. B 65, 205413 (2002).

${ }^{33}$ D. A. G. Bruggeman, Ann. Phys. 24, 636 (1935).

${ }^{34}$ Handbook of Optical Constants of Solids, edited by E. D. Palik (Academic, New York, 1985).

${ }^{35}$ B. A. Kalinikos, in Linear and Nonlinear Spin Waves in Magnetic Films and Superlattices, edited by M. G. Cottam (World Scientific, Singapore, 1994), pp. 90-156.

${ }^{36}$ M. Buchmeier, H. Dassow, D. E. Bürgler, and C. M. Schneider, Phys. Rev. B 75, 184436 (2007).

${ }^{37}$ C. Cohen-Tannoudji, Lectures on Theoretical Physics (College de France, Paris, 1964).

${ }^{38}$ Y. Roussigné, F. Ganot, C. Dugautier, P. Moch, and D. Renard, Phys. Rev. B 52, 350 (1995).

${ }^{39}$ R. E. Camley and D. L. Mills, Phys. Rev. B 18, 4821 (1978).

${ }^{40}$ M. G. Cottam, J. Phys. C 12, 1709 (1979).

${ }^{41}$ R. E. Camley, T. S. Rahman, and D. L. Mills, Phys. Rev. B 23, 1226 (1981).

${ }^{42}$ P. Grünberg, in Light Scattering from Spin-Waves in Thin Films and Layered Magnetic Structures, Light Scattering in Solids Vol. 5, edited by M. Cordona and G. Güntherrodt (Springer-Verlag, Berlin, 1989), p. 303.

${ }^{43}$ J. C. Slonczewski, Phys. Rev. B 39, 6995 (1989).

${ }^{44}$ P. Bruno, Phys. Rev. B 49, 13231 (1994).

${ }^{45}$ J. Faure-Vincent, C. Tuisan, C. Bellouard, E. Popova, M. Hehn, F. Montagne, and A. Schuhl, Phys. Rev. Lett. 89, 107206 (2002).

${ }^{46}$ J. F. Hu, V. Ng, J. P. Wang, and T. C. Chong, J. Magn. Magn. Mater. 268, 114 (2004)

${ }^{47}$ A. Butera, J. N. Zhou, and J. A. Barnard, J. Appl. Phys. 87, 5627 (2000).

${ }^{48}$ M. Vohl, J. Barnas, and P. Grünberg, Phys. Rev. B 39, 12003 (1989).

${ }^{49}$ S. Rohart, V. Repain, A. Thiaville, and S. Rousset, Phys. Rev. B 76, 104401 (2007).

${ }^{50}$ F. Wegelin, D. Valdaitsev, A. Krasiuk, S. A. Nepijko, G. Schönhense, H. J. Elmers, I. Krug, and C. M. Schnieder, Phys. Rev. B 76, 134410 (2007).

${ }^{51}$ A. A. Timopheev and S. M. Ryabchenko, Ukr. J. Phys. 53, 261 (2008).

${ }^{52}$ M. W. Müller, Phys. Rev. 122, 1485 (1961).

${ }^{53}$ F. Dumas-Bouchiat, H. S. Nagaraja, F. Rossignol, C. Champeaux, G. Trolliard, A. Catherinot, and D. Givord, J. Appl. Phys. 100, 064304 (2006). 
Journal of Applied Physics is copyrighted by the American Institute of Physics (AIP). Redistribution of journal material is subject to the AIP online journal license and/or AIP copyright. For more information, see http://ojps.aip.org/japo/japcr/jsp 